Proceedings of the Edinburgh Mathematical Society (2002) 45, 523-546 (C)

DOI:10.1017/S0013091500001097 Printed in the United Kingdom

\title{
MAXIMAL IDEALS IN THE ALGEBRA OF OPERATORS ON CERTAIN BANACH SPACES
}

\author{
NIELS JAKOB LAUSTSEN \\ Department of Pure Mathematics, University of Leeds, \\ Leeds LS2 9JT, UK (laustsen@amsta.leeds.ac.uk) \\ Present address: Department of Mathematics, University of Copenhagen, \\ Universitetsparken 5, DK-2100 Copenhagen Ø, \\ Denmark (laustsen@math.ku.dk)
}

(Received 1 November 2000)

\begin{abstract}
For a Banach space $\mathfrak{X}$, let $\mathcal{B}(\mathfrak{X})$ denote the Banach algebra of all continuous linear operators on $\mathfrak{X}$. First, we study the lattice of closed ideals in $\mathcal{B}\left(\mathfrak{J}_{p}\right)$, where $1<p<\infty$ and $\mathfrak{J}_{p}$ is the $p$ th James space. Our main result is that the ideal of weakly compact operators is the unique maximal ideal in $\mathcal{B}\left(\mathfrak{J}_{p}\right)$. Applications of this result include the following.

(i) The Brown-McCoy radical of $\mathcal{B}(\mathfrak{X})$, which by definition is the intersection of all maximal ideals in $\mathcal{B}(\mathfrak{X})$, cannot be turned into an operator ideal. This implies that there is no 'Brown-McCoy' analogue of Pietsch's construction of the operator ideal of inessential operators from the Jacobson radical of $\mathcal{B}(\mathfrak{X}) / \mathcal{A}(\mathfrak{X})$.

(ii) For each natural number $n$ and each $n$-tuple $\left(m_{1}, \ldots, m_{n}\right)$ in $\left\{k^{2} \mid k \in \mathbb{N}\right\} \cup\{\infty\}$, there is a Banach space $\mathfrak{X}$ such that $\mathcal{B}(\mathfrak{X})$ has exactly $n$ maximal ideals, and these maximal ideals have codimensions $m_{1}, \ldots, m_{n}$ in $\mathcal{B}(\mathfrak{X})$, respectively; the Banach space $\mathfrak{X}$ is a finite direct sum of James spaces and $\ell_{p}$-spaces.

Second, building on the work of Gowers and Maurey, we obtain further examples of Banach spaces $\mathfrak{X}$ such that all the maximal ideals in $\mathcal{B}(\mathfrak{X})$ can be classified. We show that the ideal of strictly singular operators is the unique maximal ideal in $\mathcal{B}(\mathfrak{X})$ for each hereditarily indecomposable Banach space $\mathfrak{X}$, and we prove that there are $2^{2^{\aleph_{0}}}$ distinct maximal ideals in $\mathcal{B}(\mathfrak{G})$, where $\mathfrak{G}$ is the Banach space constructed by Gowers to solve Banach's hyperplane problem.
\end{abstract}

Keywords: operators on Banach spaces; Banach algebras; maximal ideals; James space

AMS 2000 Mathematics subject classification: Primary 47D30; 47D50; 46H10; 16D30

\section{Introduction}

The first result concerning ideals in the Banach algebra of continuous linear operators on an infinite-dimensional Banach space is due to Calkin, who in [3] classified all the ideals in $\mathcal{B}\left(\ell_{2}\right)$. In particular he proved that the ideal of compact operators is the only non-trivial, closed ideal in $\mathcal{B}\left(\ell_{2}\right)$. For a non-separable Hilbert space $\mathfrak{H}$, Gramsch and Luft have independently described all the closed ideals in $\mathcal{B}(\mathfrak{H})$ and shown that they 
are totally ordered by inclusion (see $[\mathbf{1 3}]$ and $[\mathbf{2 2}]$, respectively). Another extension of Calkin's result is as follows.

Theorem 1.1 (Gohberg, Markus and Feldman [8]). For $\mathfrak{X}=\ell_{p}$, where $1 \leqslant p<$ $\infty$, and $\mathfrak{X}=c_{0}$, the ideal of compact operators is the unique non-trivial, closed ideal in $\mathcal{B}(\mathfrak{X})$.

We are not aware of any other infinite-dimensional Banach spaces $\mathfrak{X}$ for which the lattice of closed ideals in $\mathcal{B}(\mathfrak{X})$ is completely understood.

The aim of this paper is to study the maximal ideals in $\mathcal{B}(\mathfrak{X})$ for various Banach spaces $\mathfrak{X}$ and in this way gain insight into the closed ideal structure of $\mathcal{B}(\mathfrak{X})$. In $\S 2$ we set up our basic notation and conventions, and in $\S 3$ we outline the material from the theory of operator ideals that we require. The main result of $\S 4$ is that the ideal of weakly compact operators is the unique maximal ideal in $\mathcal{B}\left(\mathfrak{J}_{p}\right)$, where $1<p<\infty$ and $\mathfrak{J}_{p}$ is the $p$ th James space. The proof of this theorem also provides new information about the lattice of closed ideals in $\mathcal{B}\left(\mathfrak{J}_{p}\right)$; it relies on generalizations of results known for James's original space $\mathfrak{J}_{2}$. As a consequence of the uniqueness of the maximal ideal in $\mathcal{B}\left(\mathfrak{J}_{p}\right)$, we derive in $\S 5$ that the Brown-McCoy radical of $\mathcal{B}(\mathfrak{X})$, which by definition is the intersection of all maximal ideals in $\mathcal{B}(\mathfrak{X})$, cannot be turned into an operator ideal. This implies that the BrownMcCoy radical cannot be used to obtain an analogue of Pietsch's construction of the operator ideal of inessential operators from the Jacobson radical of $\mathcal{B}(\mathfrak{X}) / \mathcal{A}(\mathfrak{X})$. In $\S 6$ we study the lattice of closed ideals in $\mathcal{B}(\mathfrak{X})$ for the Banach space

$$
\mathfrak{X}:=\mathfrak{J}_{p_{1}}^{\oplus \nu_{1}} \oplus \mathfrak{J}_{p_{2}}^{\oplus \nu_{2}} \oplus \cdots \oplus \mathfrak{J}_{p_{n}}^{\oplus \nu_{n}} \oplus \ell_{p_{n+1}} \oplus \ell_{p_{n+2}} \oplus \cdots \oplus \ell_{p_{n+m}},
$$

where $n, m, \nu_{1}, \ldots, \nu_{n}$ are natural numbers and $p_{1}, \ldots, p_{n+m}$ are distinct real numbers with $\left.p_{1}, \ldots, p_{n} \in\right] 1, \infty\left[\right.$ and $p_{n+1}, \ldots, p_{n+m} \in[1, \infty[$. We show that the closed ideals in $\mathcal{B}(\mathfrak{X})$ containing the ideal of inessential operators are classified by the non-zero, closed ideals in $\mathcal{B}\left(\mathfrak{J}_{p_{1}}^{\oplus \nu_{1}}\right), \ldots, \mathcal{B}\left(\mathfrak{J}_{p_{n}}^{\oplus \nu_{n}}\right), \mathcal{B}\left(\ell_{p_{n+1}}\right), \ldots, \mathcal{B}\left(\ell_{p_{n+m}}\right)$. In particular $\mathcal{B}(\mathfrak{X})$ has exactly $n+m$ maximal ideals; $n$ of these maximal ideals have finite codimensions $\nu_{1}^{2}, \ldots, \nu_{n}^{2}$ in $\mathcal{B}(\mathfrak{X})$, respectively, and $m$ have infinite codimension in $\mathcal{B}(\mathfrak{X})$. In $\S 7$ we prove that the ideal of strictly singular operators is the unique maximal ideal in $\mathcal{B}(\mathfrak{X})$ for each hereditarily indecomposable Banach space $\mathfrak{X}$. Finally, in $\S 8$ we provide an example of a Banach space $\mathfrak{G}$ such that there are infinitely many $\left(2^{2^{\aleph_{0}}}\right.$, to be precise) distinct maximal ideals in $\mathcal{B}(\mathfrak{G})$, and we classify these maximal ideals.

\section{Notation}

Throughout this paper all vector spaces and algebras are assumed to be over the field $\mathbb{C}$ of complex numbers.

For vector spaces $\mathfrak{X}$ and $\mathfrak{Y}$, the collection of all linear maps from $\mathfrak{X}$ to $\mathfrak{Y}$ is denoted by $\mathcal{L}(\mathfrak{X}, \mathfrak{Y})$ (or $\mathcal{L}(\mathfrak{X})$ in the case where $\mathfrak{X}=\mathfrak{Y})$. We write $I_{\mathfrak{X}}$ for the identity map on $\mathfrak{X}$. Let $T: \mathfrak{X} \rightarrow \mathfrak{Y}$ be a linear map. We denote by $\operatorname{im} T$ the image of $T$ and by $\operatorname{ker} T$ the kernel of $T$. Suppose that $\mathfrak{X}_{0}$ and $\mathfrak{Y}_{0}$ are linear subspaces of $\mathfrak{X}$ and $\mathfrak{Y}$, respectively, satisfying $T\left(\mathfrak{X}_{0}\right) \subseteq \mathfrak{Y}_{0}$. Then we define $\left.T\right|_{\mathfrak{X}_{0}} ^{\mathfrak{Y}_{0}}: x \mapsto T x, \mathfrak{X}_{0} \rightarrow \mathfrak{Y}_{0}$. If $\mathfrak{X}_{0}=\mathfrak{X}$, then we omit the subscript $\mathfrak{X}_{0}$, and if $\mathfrak{Y}_{0}=\mathfrak{Y}$, then we omit the superscript $\mathfrak{Y}_{0}$. 
Let $\mathfrak{X}$ and $\mathfrak{Y}$ be Banach spaces. A continuous linear map from $\mathfrak{X}$ to $\mathfrak{Y}$ is termed an operator. The collection of all operators from $\mathfrak{X}$ to $\mathfrak{Y}$ is denoted by $\mathcal{B}(\mathfrak{X}, \mathfrak{Y})$ (or $\mathcal{B}(\mathfrak{X})$ in the case where $\mathfrak{X}=\mathfrak{Y})$. We write span $\mathfrak{X}_{0}$ for the linear span of a subset $\mathfrak{X}_{0}$ of $\mathfrak{X}$; $\overline{\text { span }} \mathfrak{X}_{0}$ denotes its norm closure. We write $\mathfrak{X}^{*}$ for the dual space of $\mathfrak{X}$, and we denote by $\kappa_{\mathfrak{X}}: \mathfrak{X} \rightarrow \mathfrak{X}^{* *}$ the canonical embedding of $\mathfrak{X}$ into its bidual space $\mathfrak{X}^{* *}$.

Let $n$ be a natural number. The direct sum of the vector spaces $\mathfrak{X}_{1}, \ldots, \mathfrak{X}_{n}$ is denoted by $\mathfrak{X}_{1} \oplus \cdots \oplus \mathfrak{X}_{n}$ (or $\mathfrak{X}^{\oplus n}$ in the case where $\mathfrak{X}_{1}=\cdots=\mathfrak{X}_{n}=\mathfrak{X}$ ). For each $k \in\{1, \ldots, n\}$, we write $J_{k}: \mathfrak{X}_{k} \rightarrow \mathfrak{X}_{1} \oplus \cdots \oplus \mathfrak{X}_{n}$ for the canonical $k$ th coordinate embedding, and we write $Q_{k}: \mathfrak{X}_{1} \oplus \cdots \oplus \mathfrak{X}_{n} \rightarrow \mathfrak{X}_{k}$ for the canonical $k$ th coordinate projection. In the case where $\mathfrak{X}_{1}, \ldots, \mathfrak{X}_{n}$ are Banach spaces, their direct sum is a Banach space for the norm $\left\|\left(x_{1}, \ldots, x_{n}\right)\right\|:=\max \left\{\left\|x_{1}\right\|, \ldots,\left\|x_{n}\right\|\right\}$, where $\left(x_{1}, \ldots, x_{n}\right) \in \mathfrak{X}_{1} \oplus \cdots \oplus \mathfrak{X}_{n}$.

Let $m$ and $n$ be natural numbers, and let $\mathfrak{X}_{1}, \ldots, \mathfrak{X}_{n}$ and $\mathfrak{Y}_{1}, \ldots, \mathfrak{Y}_{m}$ be vector spaces. With a linear map $T: \mathfrak{X}_{1} \oplus \cdots \oplus \mathfrak{X}_{n} \rightarrow \mathfrak{Y}_{1} \oplus \cdots \oplus \mathfrak{Y}_{m}$, we associate the $(m \times n)$-matrix $\left(T_{j, k}\right)_{j, k=1}^{m, n}$, where $T_{j, k}$ is the linear map given by the commutative diagram

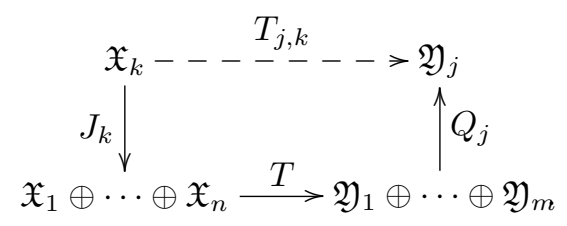

In this way we obtain a bijective correspondence between $\mathcal{L}\left(\mathfrak{X}_{1} \oplus \cdots \oplus \mathfrak{X}_{n}, \mathfrak{Y}_{1} \oplus\right.$ $\left.\cdots \oplus \mathfrak{Y}_{m}\right)$ and the set of $(m \times n)$-matrices with their $(j, k)$ th entry belonging to $\mathcal{L}\left(\mathfrak{X}_{k}, \mathfrak{Y}_{j}\right)$ for each pair $(j, k) \in\{1, \ldots, m\} \times\{1, \ldots, n\}$. This correspondence is linear, and, when defined, the composition of linear maps corresponds to the multiplication of matrices. In the case where $\mathfrak{X}_{1}, \ldots, \mathfrak{X}_{n}$ and $\mathfrak{Y}_{1}, \ldots, \mathfrak{Y}_{m}$ are Banach spaces, the subspace $\mathcal{B}\left(\mathfrak{X}_{1} \oplus \cdots \oplus \mathfrak{X}_{n}, \mathfrak{Y}_{1} \oplus \cdots \oplus \mathfrak{Y}_{m}\right)$ of $\mathcal{L}\left(\mathfrak{X}_{1} \oplus \cdots \oplus \mathfrak{X}_{n}, \mathfrak{Y}_{1} \oplus \cdots \oplus \mathfrak{Y}_{m}\right)$ is mapped onto the set of $(m \times n)$-matrices with their $(j, k)$ th entry in $\mathcal{B}\left(\mathfrak{X}_{k}, \mathfrak{Y}_{j}\right)$ for each pair $(j, k) \in\{1, \ldots, m\} \times\{1, \ldots, n\}$.

For an algebra $\mathcal{A}$ and a natural number $n$, we denote by $M_{n}(\mathcal{A})$ the algebra of $(n \times n)$ matrices over $\mathcal{A}$. The above results show that we may identify $\mathcal{L}\left(\mathfrak{X}^{\oplus n}\right)$ with $M_{n}(\mathcal{L}(\mathfrak{X}))$ for each vector space $\mathfrak{X}$. In the case where $\mathfrak{X}$ is a Banach space, this implies identifying $\mathcal{B}\left(\mathfrak{X}^{\oplus n}\right)$ with $M_{n}(\mathcal{B}(\mathfrak{X}))$.

\section{Preliminaries on operator ideals}

This section summarizes the parts of the vast theory of operator ideals that we require.

We begin with the fundamental definition which is due to Pietsch.

Definition 3.1. An operator ideal is an assignment $\mathcal{I}$ which associates with each pair $(\mathfrak{X}, \mathfrak{Y})$ of Banach spaces a linear subspace $\mathcal{I}(\mathfrak{X}, \mathfrak{Y})$ of $\mathcal{B}(\mathfrak{X}, \mathfrak{Y})$ satisfying

(i) $\mathcal{I}(\mathfrak{X}, \mathfrak{Y})$ is non-zero for some Banach spaces $\mathfrak{X}$ and $\mathfrak{Y}$; 
(ii) for any Banach spaces $\mathfrak{W}, \mathfrak{X}, \mathfrak{Y}$ and $\mathfrak{Z}$, the composite operator TSR belongs to $\mathcal{I}(\mathfrak{W}, \mathfrak{Z})$ whenever $R$ belongs to $\mathcal{B}(\mathfrak{W}, \mathfrak{X}), S$ to $\mathcal{I}(\mathfrak{X}, \mathfrak{Y})$ and $T$ to $\mathcal{B}(\mathfrak{Y}, \mathfrak{Z})$.

We usually write $\mathcal{I}(\mathfrak{X})$ instead of $\mathcal{I}(\mathfrak{X}, \mathfrak{X})$.

An operator ideal $\mathcal{I}$ is closed if the subspace $\mathcal{I}(\mathfrak{X}, \mathfrak{Y})$ is closed in the operator norm of $\mathcal{B}(\mathfrak{X}, \mathfrak{Y})$ for each pair $(\mathfrak{X}, \mathfrak{Y})$ of Banach spaces.

Remark 3.2. Operator ideals fit nicely into the matrix representation of operators between direct sums of Banach spaces described in $\S 2$. Indeed, for an operator ideal $\mathcal{I}$, Banach spaces $\mathfrak{X}_{1}, \ldots, \mathfrak{X}_{n}, \mathfrak{Y}_{1}, \ldots, \mathfrak{Y}_{m}$, and an operator $T: \mathfrak{X}_{1} \oplus \cdots \oplus \mathfrak{X}_{n} \rightarrow \mathfrak{Y}_{1} \oplus \cdots \oplus \mathfrak{Y}_{m}$ with associated matrix $\left(T_{j, k}\right)_{j, k=1}^{m, n}$, we have: $T$ belongs to $\mathcal{I}\left(\mathfrak{X}_{1} \oplus \cdots \oplus \mathfrak{X}_{n}, \mathfrak{Y}_{1} \oplus \cdots \oplus \mathfrak{Y}_{m}\right)$ if and only if $T_{j, k}$ belongs to $\mathcal{I}\left(\mathfrak{X}_{k}, \mathfrak{Y}_{j}\right)$ for each pair $(j, k) \in\{1, \ldots, m\} \times\{1, \ldots, n\}$.

In this paper, we shall consider the following standard closed operator ideals:

$\mathcal{A}$ the approximable operators;

$\mathcal{K}$ the compact operators;

$\mathcal{S}$ the strictly singular operators;

$\mathcal{E} \quad$ the inessential operators (an operator $T: \mathfrak{X} \rightarrow \mathfrak{Y}$ is termed inessential if $I_{\mathfrak{X}}-S T$ is a Fredholm operator for each operator $S: \mathfrak{Y} \rightarrow \mathfrak{X}$ );

$\mathcal{V}$ the completely continuous operators;

$\mathcal{W}$ the weakly compact operators.

For each pair $(\mathfrak{X}, \mathfrak{Y})$ of Banach spaces, the hierarchy among these operator ideals is as follows (see [23, p. 93] and [24, $\S \S 1.11$ and 26.7]):

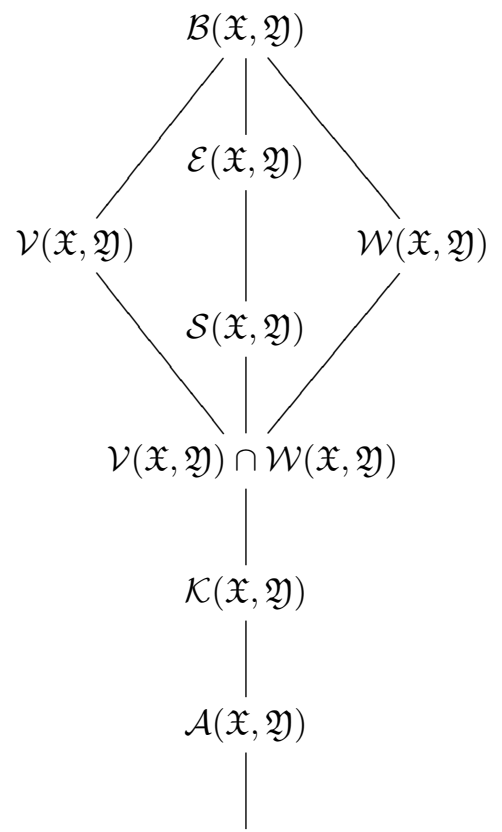

$\{0\}$. 
In this diagram, a line denotes set-theoretic inclusion with the larger set at the top of the line. All the indicated inclusions may be proper.

We shall also consider operator ideals consisting of the operators that factor through a given operator. Indeed, let $\mathfrak{E}, \mathfrak{F}, \mathfrak{X}$ and $\mathfrak{Y}$ be Banach spaces, let $\Gamma: \mathfrak{E} \rightarrow \mathfrak{F}$ be an operator, and set

$$
\mathcal{P}_{\Gamma}(\mathfrak{X}, \mathfrak{Y}):=\operatorname{span}\{T \Gamma S \mid S \in \mathcal{B}(\mathfrak{X}, \mathfrak{E}), T \in \mathcal{B}(\mathfrak{F}, \mathfrak{Y})\} \quad \text { and } \quad \mathcal{Q}_{\Gamma}(\mathfrak{X}, \mathfrak{Y}):=\overline{\mathcal{P}_{\Gamma}(\mathfrak{X}, \mathfrak{Y})} \text {. }
$$

Proposition 3.3. Let $\mathfrak{E}$ and $\mathfrak{F}$ be Banach spaces, and let $\Gamma: \mathfrak{E} \rightarrow \mathfrak{F}$ be a non-zero operator. Then $\mathcal{P}_{\Gamma}$ is an operator ideal, and $\mathcal{Q}_{\Gamma}$ is a closed operator ideal.

We call $\mathcal{P}_{\Gamma}$ the algebraic operator ideal generated by $\Gamma$ and $\mathcal{Q}_{\Gamma}$ the closed operator ideal generated by $\Gamma$. These operator ideals generalize a construction due to Porta (see $[\mathbf{2 5}]$ and $[\mathbf{2}])$. In our application we shall require the following technical fact.

Proposition 3.4. Let $\mathfrak{E}, \mathfrak{F}$ and $\mathfrak{X}$ be Banach spaces, and let $\Gamma: \mathfrak{E} \rightarrow \mathfrak{F}$ be an operator. An idempotent operator belongs to $\mathcal{P}_{\Gamma}(\mathfrak{X})$ if and only if it belongs to $\mathcal{Q}_{\Gamma}(\mathfrak{X})$.

Proof. Suppose that $P \in \mathcal{Q}_{\Gamma}(\mathfrak{X})$ is idempotent. If $P=0$, then the result is obvious. Otherwise we can take an operator $S \in \mathcal{P}_{\Gamma}(\mathfrak{X})$ such that $\|P-S\|<\|P\|^{-2}$. It follows that $\|P-P S P\|<1$. Since $P \mathcal{B}(\mathfrak{X}) P$ is a Banach algebra with identity $P$, this implies that $P S P$ is invertible in $P \mathcal{B}(\mathfrak{X}) P$ by $[\mathbf{4}$, Proposition 2.1.1], and so we can take $T \in P \mathcal{B}(\mathfrak{X}) P$ such that $(P S P) T=P$. This shows that $P$ belongs to $\mathcal{P}_{\Gamma}(\mathfrak{X})$.

For an operator $\Gamma: \mathfrak{E} \rightarrow \mathfrak{F}$, denote by $\Gamma \oplus \Gamma: \mathfrak{E} \oplus \mathfrak{E} \rightarrow \mathfrak{F} \oplus \mathfrak{F}$ the diagonal operator induced by $\Gamma$, that is, $(\Gamma \oplus \Gamma)(x, y)=(\Gamma x, \Gamma y)$ for $(x, y) \in \mathfrak{E} \oplus \mathfrak{E}$. An immediate generalization of the proof of $[\mathbf{2}$, Theorem 5.13] yields the following proposition.

Proposition 3.5. Let $\mathfrak{E}$ and $\mathfrak{F}$ be Banach spaces. For each operator $\Gamma: \mathfrak{E} \rightarrow \mathfrak{F}$, the following assertions are equivalent:

(a) there are operators $U: \mathfrak{E} \oplus \mathfrak{E} \rightarrow \mathfrak{E}$ and $V: \mathfrak{F} \rightarrow \mathfrak{F} \oplus \mathfrak{F}$ such that $\Gamma \oplus \Gamma=V \Gamma U$; and

(b) $\mathcal{P}_{\Gamma}(\mathfrak{X}, \mathfrak{Y})=\{T \Gamma S \mid S \in \mathcal{B}(\mathfrak{X}, \mathfrak{E}), T \in \mathcal{B}(\mathfrak{F}, \mathfrak{Y})\}$ for each pair $(\mathfrak{X}, \mathfrak{Y})$ of Banach spaces.

Lemma 3.6. Let $\mathfrak{X}$ and $\mathfrak{Y}$ be Banach spaces.

(i) Let $P: \mathfrak{X} \rightarrow \mathfrak{X}$ and $Q: \mathfrak{Y} \rightarrow \mathfrak{Y}$ be idempotent operators. There are operators $S: \mathfrak{X} \rightarrow \mathfrak{Y}$ and $T: \mathfrak{Y} \rightarrow \mathfrak{X}$ such that $P=T S$ and $Q=S T$ if and only if the images of $P$ and $Q$ are isomorphic.

(ii) Suppose that $S: \mathfrak{X} \rightarrow \mathfrak{Y}$ and $T: \mathfrak{Y} \rightarrow \mathfrak{X}$ are operators such that $T S$ is idempotent. Then STST is idempotent, and the images of TS and STST are isomorphic.

Proof. (i) If $S: \mathfrak{X} \rightarrow \mathfrak{Y}$ and $T: \mathfrak{Y} \rightarrow \mathfrak{X}$ are operators with $P=T S$ and $Q=S T$, then $S(\operatorname{im} P) \subseteq \operatorname{im} Q, T(\operatorname{im} Q) \subseteq \operatorname{im} P$, and $\left.S\right|_{\operatorname{im} P} ^{\operatorname{im} Q}$ is an isomorphism with inverse $\left.T\right|_{\operatorname{im} Q} ^{\operatorname{im} P}$.

Conversely, if $U: \operatorname{im} P \rightarrow \operatorname{im} Q$ is an isomorphism, then the operators $S:=\left.\left.U\right|^{\mathfrak{Y}} P\right|^{\operatorname{im} P}$ and $T:=\left.\left.\left(U^{-1}\right)\right|^{\mathfrak{x}} Q\right|^{\mathrm{im} Q}$ satisfy $T S=P$ and $S T=Q$.

(ii) This is immediate from (i). 
In the case where the generating operator $\Gamma$ is idempotent, we can now reformulate Proposition 3.5 as follows.

Corollary 3.7. For an idempotent operator $\Gamma$ on a Banach space $\mathfrak{E}$, the following assertions are equivalent:

(a) $\operatorname{im} \Gamma$ contains a complemented subspace isomorphic to $\operatorname{im} \Gamma \oplus \operatorname{im} \Gamma$; and

(b) $\mathcal{P}_{\Gamma}(\mathfrak{X}, \mathfrak{Y})=\{T S \mid S \in \mathcal{B}(\mathfrak{X}, \operatorname{im} \Gamma), T \in \mathcal{B}(\operatorname{im} \Gamma, \mathfrak{Y})\}$ for each pair $(\mathfrak{X}, \mathfrak{Y})$ of Banach spaces.

\section{The lattice of closed ideals in $\mathcal{B}\left(\mathfrak{J}_{p}\right)$ for $1<p<\infty$}

Throughout this section, we fix a real number $p$ in the open interval $] 1, \infty[$. Our aim is to understand the lattice of closed ideals in $\mathcal{B}\left(\mathfrak{J}_{p}\right)$, where $\mathfrak{J}_{p}$ is the $p$ th James space (to be defined below). Our main result is that $\mathcal{W}\left(\mathfrak{J}_{p}\right)$ is the unique maximal ideal in $\mathcal{B}\left(\mathfrak{J}_{p}\right)$.

For $p=2$, Loy and Willis have shown that $\mathcal{K}\left(\mathfrak{J}_{2}\right) \subsetneq \mathcal{Q}_{\ell_{\ell_{2}}}\left(\mathfrak{J}_{2}\right) \subsetneq \mathcal{W}\left(\mathfrak{J}_{2}\right)$ and that $\mathcal{Q}_{I_{\ell_{2}}}\left(\mathfrak{J}_{2}\right) \nsubseteq \mathcal{S}\left(\mathfrak{J}_{2}\right)=\mathcal{E}\left(\mathfrak{J}_{2}\right)$ (see [21, Theorem 2.7]). Saksman and Tylli have improved this by observing that $\mathcal{K}\left(\mathfrak{J}_{2}\right)=\mathcal{S}\left(\mathfrak{J}_{2}\right)$ (see $[\mathbf{2 6}$, Remark 3.9]). We generalize these results to arbitrary $p \in] 1, \infty[$, and we complement them, showing that the lattice of closed ideals in $\mathcal{B}\left(\mathfrak{J}_{p}\right)$ has the following structure:

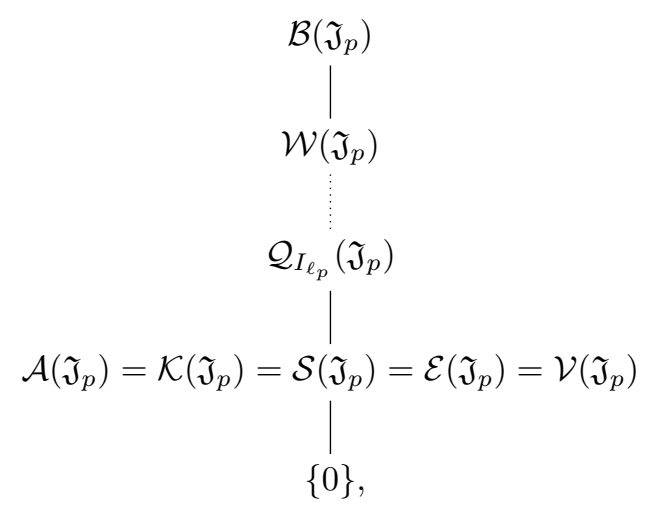

where all the indicated inclusions are proper, and only at the dotted line may there be further closed ideals.

Definition 4.1. For each sequence $x=\left(\alpha_{k}\right)_{k \in \mathbb{N}}$ of complex numbers, set

$\|x\|_{\mathfrak{J}_{p}}:=\sup \left\{\left(\sum_{m=1}^{n-1}\left|\alpha_{k_{m}}-\alpha_{k_{m+1}}\right|^{p}\right)^{1 / p} \mid n, k_{1}, \ldots, k_{n} \in \mathbb{N}, n \geqslant 2, k_{1}<k_{2}<\cdots<k_{n}\right\}$. 
The pth James space is

$$
\mathfrak{J}_{p}:=\left\{\left(\alpha_{k}\right)_{k \in \mathbb{N}} \mid \alpha_{k} \in \mathbb{C}(k \in \mathbb{N}),\left\|\left(\alpha_{k}\right)_{k \in \mathbb{N}}\right\|_{\mathfrak{J}_{p}}<\infty \text { and } \alpha_{k} \rightarrow 0 \text { as } k \rightarrow \infty\right\} .
$$

Then $\left(\mathfrak{J}_{p},\|\cdot\|_{\mathfrak{J}_{p}}\right)$ is a Banach space with a monotone basis $\left(e_{k}\right)_{k \in \mathbb{N}}$ given by $e_{k}=$ $\left(\delta_{k, m}\right)_{m \in \mathbb{N}}$ for each $k \in \mathbb{N}$, where $\delta_{k, m}$ is Kronecker's delta symbol. This basis is shrinking, so that the biorthogonal functionals $\left(f_{k}\right)_{k \in \mathbb{N}}$ associated with $\left(e_{k}\right)_{k \in \mathbb{N}}$ form a basis for $\mathfrak{J}_{p}^{*}$.

A fundamental property of $\mathfrak{J}_{p}$ is that it is quasireflexive, that is, its canonical image $\kappa_{\mathfrak{J}_{p}}\left(\mathfrak{J}_{p}\right)$ in its bidual space $\mathfrak{J}_{p}^{* *}$ has codimension one; for $p=2$, this is due to James (see [16]). An immediate consequence of the quasireflexivity of $\mathfrak{J}_{p}$ is that the ideal $\mathcal{W}\left(\mathfrak{J}_{p}\right)$ of weakly compact operators has codimension one in $\mathcal{B}\left(\mathfrak{J}_{p}\right)$ and hence is a maximal ideal.

We shall now introduce a class of operators on $\mathfrak{J}_{p}$ which will play an important role in our analysis of the closed ideal structure of $\mathcal{B}\left(\mathfrak{J}_{p}\right)$. Set

$$
\mathbb{J}:=\left\{\left(j_{k}\right)_{k \in \mathbb{N}_{0}} \mid j_{0}=0, j_{k} \in \mathbb{N} \text { and } j_{k-1}<j_{k}(k \in \mathbb{N})\right\},
$$

and, for each $\boldsymbol{j}=\left(j_{k}\right)_{k \in \mathbb{N}_{0}} \in \mathbb{J}$, define

$$
H_{j} e_{k}:=\sum_{m=j_{k-1}+1}^{j_{k}} e_{m} \quad \text { and } \quad G_{j} e_{k}:=\left\{\begin{array}{ll}
e_{m} & \text { if } k=j_{m} \text { for some } m \in \mathbb{N}, \\
0 & \text { otherwise }
\end{array} \quad(k \in \mathbb{N})\right.
$$

By linearity and continuity, $H_{\boldsymbol{j}}$ and $G_{\boldsymbol{j}}$ extend to operators (also denoted by $H_{\boldsymbol{j}}$ and $G_{\boldsymbol{j}}$ ) of norm one on $\mathfrak{J}_{p}$ satisfying

$$
G_{\boldsymbol{j}} H_{\boldsymbol{j}}=I_{\mathfrak{J}_{p}}
$$

It follows that $H_{\boldsymbol{j}} G_{\boldsymbol{j}}$ and hence $P_{\boldsymbol{j}}:=I_{\mathfrak{J}_{p}}-H_{\boldsymbol{j}} G_{\boldsymbol{j}}$ are idempotent operators.

Lemma 4.2. Let $\boldsymbol{j} \in \mathbb{J}$. The kernel of $P_{\boldsymbol{j}}$ is given by

$$
\operatorname{ker} P_{\boldsymbol{j}}=\operatorname{im} H_{\boldsymbol{j}}=\left\{\sum_{k=1}^{\infty} \alpha_{k}\left(\sum_{m=j_{k-1}+1}^{j_{k}} e_{m}\right) \mid\left(\alpha_{k}\right)_{k \in \mathbb{N}} \in \mathfrak{J}_{p}\right\}
$$

and it is isomorphic to $\mathfrak{J}_{p}$. The image of $P_{\boldsymbol{j}}$ is given by

$$
\operatorname{im} P_{\boldsymbol{j}}=\operatorname{ker} G_{\boldsymbol{j}}=\overline{\operatorname{span}}\left\{e_{k} \mid k \in \mathbb{N} \backslash\left\{j_{m} \mid m \in \mathbb{N}\right\}\right\},
$$

and it is reflexive.

Proof. The identities (4.3) and (4.4) are easily verified using (4.2). Lemma 3.6 (i) implies that $\operatorname{ker} P_{\boldsymbol{j}}=\operatorname{im} H_{\boldsymbol{j}} G_{\boldsymbol{j}} \cong \operatorname{im} G_{\boldsymbol{j}} H_{\boldsymbol{j}}=\mathfrak{J}_{p}$. Since $\operatorname{ker} P_{\boldsymbol{j}} \oplus \operatorname{im} P_{\boldsymbol{j}} \cong \mathfrak{J}_{p}$, we conclude that $\operatorname{im} P_{j}$ is reflexive.

Note that Lemma 4.2 has [5, Corollary 3 and Theorem 5 (including Remark (ii))] as immediate consequences. 
To determine the isomorphism type of the complemented subspace im $P_{\boldsymbol{j}}$ of $\mathfrak{J}_{p}$ for each $j \in \mathbb{J}$, we consider, for each $n \in \mathbb{N}$, the $n$-dimensional Banach space

$$
\mathfrak{J}_{p}^{(n)}:=\operatorname{span}\left\{e_{1}, \ldots, e_{n}\right\}
$$

equipped with the norm it inherits as a subspace of $\mathfrak{J}_{p}$. Let $\left(n_{k}\right)_{k \in \mathbb{N}}$ be a sequence of natural numbers, and let $\left(\bigoplus_{k=1}^{\infty} \mathfrak{J}_{p}^{\left(n_{k}\right)}\right)_{\ell_{p}}$ denote the direct sum of $\mathfrak{J}_{p}^{\left(n_{1}\right)}, \mathfrak{J}_{p}^{\left(n_{2}\right)}, \ldots$ in the sense of $\ell_{p}$. As for finite direct sums, we write

$$
J_{k}: \mathfrak{J}_{p}^{\left(n_{k}\right)} \rightarrow\left(\bigoplus_{m=1}^{\infty} \mathfrak{J}_{p}^{\left(n_{m}\right)}\right)_{\ell_{p}}
$$

for the canonical $k$ th coordinate embedding. The canonical basis

$$
\begin{aligned}
\left(J_{1}\left(e_{1}\right), J_{1}\left(e_{2}\right), \ldots, J_{1}\left(e_{n_{1}}\right), J_{2}\left(e_{1}\right),\right. & J_{2}\left(e_{2}\right), \ldots \\
& \left.J_{2}\left(e_{n_{2}}\right), \ldots, J_{k}\left(e_{1}\right), J_{k}\left(e_{2}\right), \ldots, J_{k}\left(e_{n_{k}}\right), \ldots\right)
\end{aligned}
$$

of $\left(\bigoplus_{k=1}^{\infty} \mathfrak{J}_{p}^{\left(n_{k}\right)}\right)_{\ell_{p}}$ satisfies

$$
\left\|J_{k}\left(e_{1}\right)+J_{k}\left(e_{2}\right)+\cdots+J_{k}\left(e_{n_{k}}\right)\right\|=\left\|e_{1}+e_{2}+\cdots+e_{n_{k}}\right\|_{\mathfrak{J}_{p}}=1 \quad(k \in \mathbb{N}) .
$$

If the sequence $\left(n_{k}\right)_{k \in \mathbb{N}}$ is unbounded, then this implies that $\left(\bigoplus_{k=1}^{\infty} \mathfrak{J}_{p}^{\left(n_{k}\right)}\right)_{\ell_{p}}$ is not uniformly convexifiable by Gurariı-Gurariı̀'s Theorem (see [14, Theorems 345 and 352]); for $p=2$, this is observed in [5, p. 267]. On the other hand, Clarkson's theorem states that $\ell_{p}$ is uniformly convex (see [14, Theorem 333]), and so we reach the following conclusion.

Lemma 4.3. Let $\left(n_{k}\right)_{k \in \mathbb{N}}$ be an unbounded sequence of natural numbers. Then $\ell_{p}$ is not isomorphic to $\left(\bigoplus_{k=1}^{\infty} \mathfrak{J}_{p}^{\left(n_{k}\right)}\right)_{\ell_{p}}$.

Proposition 4.4. For each $\boldsymbol{j}=\left(j_{k}\right)_{k \in \mathbb{N}_{0}} \in \mathbb{J}$, set $\operatorname{gap}(\boldsymbol{j}):=\left\{k \in \mathbb{N} \mid j_{k}>j_{k-1}+1\right\}$.

(i) If $\operatorname{gap}(\boldsymbol{j})$ is the empty set, then $P_{\boldsymbol{j}}=0$ and $\mathcal{Q}_{P_{\boldsymbol{j}}}\left(\mathfrak{J}_{p}\right)=\{0\}$.

(ii) If $\operatorname{gap}(\boldsymbol{j})$ is a finite, non-empty set, then $P_{\boldsymbol{j}}$ has finite rank and $\mathcal{Q}_{P_{\boldsymbol{j}}}\left(\mathfrak{J}_{p}\right)=\mathcal{A}\left(\mathfrak{J}_{p}\right)$.

(iii) If $\operatorname{gap}(\boldsymbol{j})$ is an infinite set and the sequence $\left(j_{k}-j_{k-1}\right)_{k \in \mathbb{N}}$ is bounded, then the image of $P_{\boldsymbol{j}}$ is isomorphic to $\ell_{p}$ and $\mathcal{Q}_{P_{j}}\left(\mathfrak{J}_{p}\right)=\mathcal{Q}_{I_{\ell_{p}}}\left(\mathfrak{J}_{p}\right)$.

(iv) If the sequence $\left(j_{k}-j_{k-1}\right)_{k \in \mathbb{N}}$ is unbounded, then the image of $P_{\boldsymbol{j}}$ is isomorphic to $\left(\bigoplus_{n=1}^{\infty} \mathfrak{J}_{p}^{(n)}\right)_{\ell_{p}}$ and $\mathcal{Q}_{I_{\ell_{p}}}\left(\mathfrak{J}_{p}\right) \subsetneq \mathcal{Q}_{P_{j}}\left(\mathfrak{J}_{p}\right) \subseteq \mathcal{W}\left(\mathfrak{J}_{p}\right)$.

This characterization will be completed in Proposition 4.18, below, where we show that if the sequence $\left(j_{k}-j_{k-1}\right)_{k \in \mathbb{N}}$ is unbounded, then $\mathcal{Q}_{P_{\boldsymbol{j}}}\left(\mathfrak{J}_{p}\right)=\mathcal{W}\left(\mathfrak{J}_{p}\right)$. 
Proof. Clauses (i) and (ii) are obvious, so we may assume that $\operatorname{gap}(\boldsymbol{j})$ is an infinite set, say $\operatorname{gap}(\boldsymbol{j})=\left\{\nu_{1}, \nu_{2}, \ldots\right\}$, where $\nu_{1}<\nu_{2}<\cdots$. For each $k \in \mathbb{N}$, set $\mu_{k}:=j_{\nu_{k}-1} \in \mathbb{N}_{0}$ and $n_{k}:=j_{\nu_{k}}-j_{\nu_{k}-1}-1 \in \mathbb{N}$. Using (4.4), it is straightforward to verify that

$$
\operatorname{im} P_{j}=\overline{\operatorname{span}}\left\{e_{\mu_{k}+m} \mid k \in \mathbb{N}, m \in\left\{1, \ldots, n_{k}\right\}\right\} .
$$

Observe that $\mu_{k}+n_{k}<\mu_{k+1}$ for each $k \in \mathbb{N}$, so that we can define a linear map

$$
V: \operatorname{span}\left\{e_{\mu_{k}+m} \mid k \in \mathbb{N}, m \in\left\{1, \ldots, n_{k}\right\}\right\} \rightarrow\left(\bigoplus_{k=1}^{\infty} \mathfrak{J}_{p}^{\left(n_{k}\right)}\right)_{\ell_{p}}
$$

by $V e_{\mu_{k}+m}:=J_{k}\left(e_{m}\right)$ for $k \in \mathbb{N}$ and $m \in\left\{1, \ldots, n_{k}\right\}$. As in the proof of [5, Lemma 2], we can show that $V$ is continuous and bounded below. Since $V$ has dense image, it extends to an isomorphism from im $P_{\boldsymbol{j}}$ onto $\left(\bigoplus_{k=1}^{\infty} \mathfrak{J}_{p}^{\left(n_{k}\right)}\right) \ell_{p}$.

In the case where the sequence $\left(j_{k}-j_{k-1}\right)_{k \in \mathbb{N}}$ is bounded, it is clear that $\left(\bigoplus_{k=1}^{\infty} \mathfrak{J}_{p}^{\left(n_{k}\right)}\right)_{\ell_{p}}$ is isomorphic to $\ell_{p}$, and (iii) follows.

For $\left(j_{k}-j_{k-1}\right)_{k \in \mathbb{N}}$ unbounded, $\left(\bigoplus_{k=1}^{\infty} \mathfrak{J}_{p}^{\left(n_{k}\right)}\right)_{\ell_{p}}$ is isomorphic to $\left(\bigoplus_{n=1}^{\infty} \mathfrak{J}_{p}^{(n)}\right)_{\ell_{p}}$ by $[\mathbf{6}$, Lemma 5] because the Banach-Mazur distance between the $(m+n)$-dimensional Banach spaces $\mathfrak{J}_{p}^{(m+n)}$ and $\mathfrak{J}_{p}^{(m)} \oplus \mathfrak{J}_{p}^{(n)}$ is uniformly bounded in $(m, n) \in \mathbb{N}^{2}$. The inclusions $\mathcal{Q}_{I_{\ell_{p}}}\left(\mathfrak{J}_{p}\right) \subseteq \mathcal{Q}_{P_{j}}\left(\mathfrak{J}_{p}\right) \subseteq \mathcal{W}\left(\mathfrak{J}_{p}\right)$ hold because im $P_{\boldsymbol{j}}$ is reflexive and contains a complemented subspace isomorphic to $\ell_{p}$. Assume towards a contradiction that $P_{\boldsymbol{j}}$ belongs to $\mathcal{Q}_{I_{\ell_{p}}}\left(\mathfrak{J}_{p}\right)$. Then Proposition 3.4 shows that $P_{\boldsymbol{j}}$ actually belongs to $\mathcal{P}_{I_{\ell_{p}}}\left(\mathfrak{J}_{p}\right)$. By Corollary 3.7, there are operators $S: \mathfrak{J}_{p} \rightarrow \ell_{p}$ and $T: \ell_{p} \rightarrow \mathfrak{J}_{p}$ such that $P_{\boldsymbol{j}}=T S$. It follows from Lemma 3.6 (ii) that $Q:=S T S T \in \mathcal{B}\left(\ell_{p}\right)$ is idempotent and $\operatorname{im} Q$ is isomorphic to $\operatorname{im} P_{\boldsymbol{j}}$. In particular, $\operatorname{im} Q$ is infinite dimensional, so [20, Theorem 2.a.3] implies that $\operatorname{im} Q$ is isomorphic to $\ell_{p}$, contradicting Lemma 4.3.

We note in particular that Proposition 4.4 (iv) generalizes Loy and Willis's observation that $\mathcal{Q}_{I_{\ell_{2}}}\left(\mathfrak{J}_{2}\right) \subsetneq \mathcal{W}\left(\mathfrak{J}_{2}\right)$.

Corollary 4.5. The closed ideal $\mathcal{Q}_{I_{\ell_{p}}}\left(\mathfrak{J}_{p}\right)$ is properly contained in $\mathcal{W}\left(\mathfrak{J}_{p}\right)$.

For $p=2$, our next proposition is a combination of results due to Herman and Whitley (see the proof of Lemma 1 in [15]) and Casazza, Bor-Luh Lin and Lohman (see [5, Theorem 10]). The original proofs can be generalized directly; our approach using Proposition 4.4 gives a quick unified proof.

Lemma 4.6. Let $\left(x_{k}\right)_{k \in \mathbb{N}}$ be a normalized block basic sequence of the basis $\left(e_{k}\right)_{k \in \mathbb{N}}$ of $\mathfrak{J}_{p}$ with a 'gap' between any two consecutive blocks, that is, each basis vector has the form

$$
x_{k}=\sum_{m=j_{k-1}+1}^{j_{k}-1} \alpha_{m} e_{m} \quad(k \in \mathbb{N}),
$$

where $\alpha_{1}, \alpha_{2}, \ldots$ are complex numbers, $j_{0}=0$, and $j_{1}, j_{2}, \ldots$ are integers satisfying

$$
j_{k}>j_{k-1}+1 \quad(k \in \mathbb{N}) \text {. }
$$


Then $\left(x_{k}\right)_{k \in \mathbb{N}}$ is equivalent to the standard basis of $\ell_{p}$, and $\overline{\operatorname{span}}\left\{x_{k} \mid k \in \mathbb{N}\right\}$ is complemented in $\mathfrak{J}_{p}$.

Proof. By assumption, $\boldsymbol{j}:=\left(j_{k}\right)_{k \in \mathbb{N}_{0}}$ belongs to $\mathbb{J}$ with $\operatorname{gap}(\boldsymbol{j})=\mathbb{N}$. As in the proof of Proposition 4.4, we can construct an isomorphism $V: \operatorname{im} P_{\boldsymbol{j}} \rightarrow\left(\bigoplus_{k=1}^{\infty} \mathfrak{J}_{p}^{\left(n_{k}\right)}\right)_{\ell_{p}}$, where $n_{k}=j_{k}-j_{k-1}-1$ for each $k \in \mathbb{N}$. Since $\overline{\operatorname{span}}\left\{x_{k} \mid k \in \mathbb{N}\right\}$ is contained in im $P_{j}$ by (4.4) and im $P_{\boldsymbol{j}}$ is complemented in $\mathfrak{J}_{p}$, it suffices to show that the basic sequence $\left(V x_{k}\right)_{k \in \mathbb{N}}$ is equivalent to the standard basis of $\ell_{p}$ and that $\overline{\operatorname{span}}\left\{V x_{k} \mid k \in \mathbb{N}\right\}$ is complemented in $\left(\bigoplus_{k=1}^{\infty} \mathfrak{J}_{p}^{\left(n_{k}\right)}\right)_{\ell_{p}}$. However, this follows immediately from the facts that $V x_{k} \in \operatorname{im} J_{k}$ for each $k \in \mathbb{N}, \inf \left\{\left\|V x_{k}\right\| \mid k \in \mathbb{N}\right\}>0$, and $\sup \left\{\left\|V x_{k}\right\| \mid k \in \mathbb{N}\right\}<\infty$.

We can now generalize the proof of [5, Corollary 11] to obtain the following important result.

Corollary 4.7. Each closed, infinite-dimensional subspace of $\mathfrak{J}_{p}$ contains a subspace which is isomorphic to $\ell_{p}$ and complemented in $\mathfrak{J}_{p}$.

Lemma 4.8. Suppose that $\left(x_{k}\right)_{k \in \mathbb{N}}$ is a sequence in $\mathfrak{J}_{p}$ satisfying the following:

(i) $\left(x_{k}\right)_{k \in \mathbb{N}}$ converges weakly to zero; and

(ii) $\inf \left\{\left\|x_{k}\right\|_{\mathfrak{J}_{p}} \mid k \in \mathbb{N}\right\}>0$.

Then a subsequence of $\left(x_{k}\right)_{k \in \mathbb{N}}$ is a basic sequence equivalent to the standard basis of $\ell_{p}$.

Proof. By [20, Proposition 1.a.12], a subsequence $\left(x_{k}^{\prime}\right)_{k \in \mathbb{N}}$ of $\left(x_{k}\right)_{k \in \mathbb{N}}$ is equivalent to a block basic sequence $\left(w_{k}\right)_{k \in \mathbb{N}}$ of $\left(e_{k}\right)_{k \in \mathbb{N}}$. Lemma 4.6 implies that $\left(w_{2 k} /\left\|w_{2 k}\right\|_{\mathfrak{J}_{p}}\right)_{k \in \mathbb{N}}$ is equivalent to the standard basis $\left(d_{k}\right)_{k \in \mathbb{N}}$ of $\ell_{p}$. By the uniform boundedness principle, $\left(x_{k}\right)_{k \in \mathbb{N}}$ is norm-bounded, and therefore $\left(w_{k}\right)_{k \in \mathbb{N}}$ is norm-bounded. Condition (ii) implies that $\inf \left\{\left\|w_{k}\right\|_{\mathfrak{J}_{p}} \mid k \in \mathbb{N}\right\}>0$. It follows that $\left(w_{2 k}\right)_{k \in \mathbb{N}}$ is equivalent to $\left(d_{k}\right)_{k \in \mathbb{N}}$ because $\left(d_{k}\right)_{k \in \mathbb{N}}$ is an unconditional basis. Hence the subsequence $\left(x_{2 k}^{\prime}\right)_{k \in \mathbb{N}}$ of $\left(x_{k}\right)_{k \in \mathbb{N}}$ has the desired properties.

We can now generalize Loy-Willis's and Saksman-Tylli's result that $\mathcal{K}\left(\mathfrak{J}_{2}\right)=\mathcal{E}\left(\mathfrak{J}_{2}\right)$.

Proposition 4.9. The ideals of approximable, compact, strictly singular, inessential and completely continuous operators coincide in $\mathcal{B}\left(\mathfrak{J}_{p}\right)$ :

$$
\mathcal{A}\left(\mathfrak{J}_{p}\right)=\mathcal{K}\left(\mathfrak{J}_{p}\right)=\mathcal{S}\left(\mathfrak{J}_{p}\right)=\mathcal{E}\left(\mathfrak{J}_{p}\right)=\mathcal{V}\left(\mathfrak{J}_{p}\right)
$$

Proof. The fact that $\mathfrak{J}_{p}$ has a basis implies that $\mathcal{A}\left(\mathfrak{J}_{p}\right)=\mathcal{K}\left(\mathfrak{J}_{p}\right)$. Since $\mathfrak{J}_{p}^{*}$ has a basis, it is separable, and so $\mathcal{K}\left(\mathfrak{J}_{p}\right)=\mathcal{V}\left(\mathfrak{J}_{p}\right)$ by [18, Theorems 3 and 5$]$. As observed in [21, p. 334] in the case $p=2$, Corollary 4.7 implies that $\mathcal{S}\left(\mathfrak{J}_{p}\right)=\mathcal{E}\left(\mathfrak{J}_{p}\right)$ by $[\mathbf{4}$, Theorem 5.6.5]. 
Suppose that the operator $T$ on $\mathfrak{J}_{p}$ is not completely continuous. Then there is a sequence $\left(x_{k}\right)_{k \in \mathbb{N}}$ in $\mathfrak{J}_{p}$ satisfying

(i) $\left(x_{k}\right)_{k \in \mathbb{N}}$ converges weakly to zero; and

(ii) $\inf \left\{\left\|T x_{k}\right\|_{\mathfrak{J}_{p}} \mid k \in \mathbb{N}\right\}>0$.

Condition (ii) implies that $\inf \left\{\left\|x_{k}\right\|_{\mathfrak{J}_{p}} \mid k \in \mathbb{N}\right\}>0$, and so, by Lemma 4.8, we can take a subsequence $\left(x_{k}^{\prime}\right)_{k \in \mathbb{N}}$ of $\left(x_{k}\right)_{k \in \mathbb{N}}$ and an operator $U: \ell_{p} \rightarrow \mathfrak{J}_{p}$ which is bounded below and satisfies $U d_{k}=x_{k}^{\prime}$ for each $k \in \mathbb{N}$, where $\left(d_{k}\right)_{k \in \mathbb{N}}$ is the standard basis of $\ell_{p}$.

The sequence $\left(T x_{k}^{\prime}\right)_{k \in \mathbb{N}}$ converges weakly to zero by (i), and $\inf \left\{\left\|T x_{k}^{\prime}\right\|_{\mathfrak{J}_{p}} \mid k \in \mathbb{N}\right\}>0$ by (ii). Hence, another application of Lemma 4.8 yields a subsequence $\left(x_{k_{m}}^{\prime}\right)_{m \in \mathbb{N}}$ of $\left(x_{k}^{\prime}\right)_{k \in \mathbb{N}}$ and an operator $V: \ell_{p} \rightarrow \mathfrak{J}_{p}$ which is bounded below and satisfies $V d_{m}=T x_{k_{m}}^{\prime}$ for each $m \in \mathbb{N}$. The fact that $\left(d_{k}\right)_{k \in \mathbb{N}}$ is a (sub)symmetric basis implies that there is an operator $W: \ell_{p} \rightarrow \ell_{p}$ which is bounded below and satisfies $W d_{m}=d_{k_{m}}$ for each $m \in \mathbb{N}$ by $\left[\mathbf{2 0}, \S 3\right.$.a]. Since $T U W d_{m}=V d_{m}$ for each $m \in \mathbb{N}$, we have $T U W=V$, and so $T$ is not strictly singular because $V$ is not strictly singular. We have thus shown that $\mathcal{S}\left(\mathfrak{J}_{p}\right) \subseteq \mathcal{V}\left(\mathfrak{J}_{p}\right)=\mathcal{K}\left(\mathfrak{J}_{p}\right)$, and the proof is complete.

Lemma 4.10. For each non-compact operator $T$ on $\mathfrak{J}_{p}$, there is a closed subspace $\mathfrak{W}$ of $\mathfrak{J}_{p}$ satisfying

(i) $\mathfrak{W}$ is isomorphic to $\ell_{p}$;

(ii) the restriction of $T$ to $\mathfrak{W}$ is bounded below; and

(iii) $T(\mathfrak{W})$ is complemented in $\mathfrak{J}_{p}$.

Proof. By Proposition 4.9, $T$ is not strictly singular. Take a closed, infinite-dimensional subspace $\mathfrak{X}$ of $\mathfrak{J}_{p}$ such that $\left.T\right|_{\mathfrak{X}}$ is bounded below. Then $T(\mathfrak{X})$ is a closed, infinitedimensional subspace of $\mathfrak{J}_{p}$, so that Corollary 4.7 implies that $T(\mathfrak{X})$ contains a subspace $\mathfrak{Y}$ which is isomorphic to $\ell_{p}$ and complemented in $\mathfrak{J}_{p}$. Set $\mathfrak{W}:=\mathfrak{X} \cap T^{-1}(\mathfrak{Y})$. It follows that $\left.T\right|_{\mathfrak{W}} ^{\mathfrak{Y}}: \mathfrak{W} \rightarrow \mathfrak{Y}$ is an isomorphism, and hence (i)-(iii) hold.

Remark 4.11. Conditions (ii) and (iii) of Lemma 4.10 imply that the subspace $\mathfrak{W}$ is complemented in $\mathfrak{J}_{p}$ by $[\mathbf{2 8}$, Lemma 1.1].

Corollary 4.12. Each ideal in $\mathcal{B}\left(\mathfrak{J}_{p}\right)$ strictly larger than $\mathcal{K}\left(\mathfrak{J}_{p}\right)$ contains $\mathcal{P}_{I_{\ell_{p}}}\left(\mathfrak{J}_{p}\right)$.

Proof. Suppose that $\mathcal{I}$ is an ideal in $\mathcal{B}\left(\mathfrak{J}_{p}\right)$ containing a non-compact operator $T$. Then there is a closed subspace $\mathfrak{W}$ of $\mathfrak{J}_{p}$ satisfying conditions (i)-(iii) of Lemma 4.10. Take an isomorphism $U: \ell_{p} \rightarrow \mathfrak{W}$ and an idempotent operator $P$ on $\mathfrak{J}_{p}$ with $\operatorname{im} P=T(\mathfrak{W})$. Set $V:=\left(\left.T\right|_{\mathfrak{W}} ^{T(\mathfrak{W})} U\right)^{-1} \in \mathcal{B}\left(T(\mathfrak{W}), \ell_{p}\right)$. For each operator $S$ in $\mathcal{P}_{I_{\ell_{p}}}\left(\mathfrak{J}_{p}\right)$, there are operators $A: \mathfrak{J}_{p} \rightarrow \ell_{p}$ and $B: \ell_{p} \rightarrow \mathfrak{J}_{p}$ with $S=B A$ by Corollary 3.7. We define operators $C$ and 
$D$ on $\mathfrak{J}_{p}$ such that the diagram

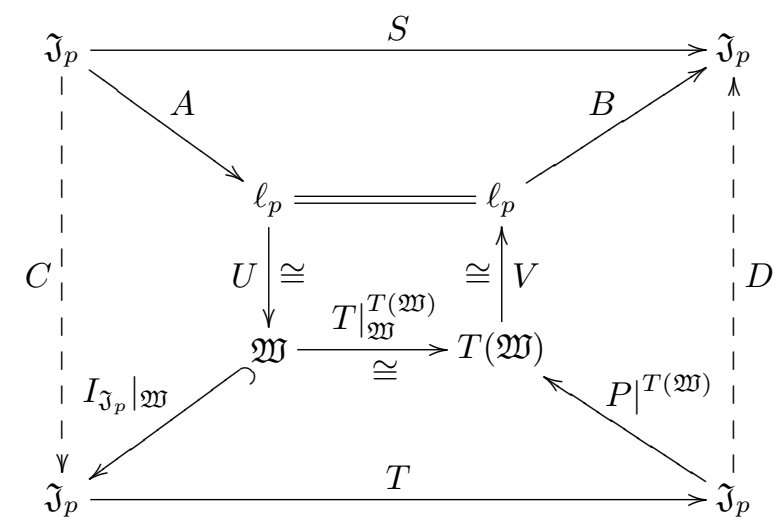

is commutative. It follows that $S$ factors through $T$, and hence $S$ belongs to $\mathcal{I}$.

With each operator $T$ on $\mathfrak{J}_{p}$, we associate the (infinite) matrix $\left(T_{k, m}\right)_{k, m=1}^{\infty}$, where

$$
T_{k, m}:=f_{k}\left(T e_{m}\right) \in \mathbb{C} \quad(k, m \in \mathbb{N}) .
$$

The facts that $\left(f_{k}\right)_{k \in \mathbb{N}}$ is a basis of $\mathfrak{J}_{p}^{*}$ and that $\sum_{m=1}^{n} e_{m}$ is a unit vector in $\mathfrak{J}_{p}$ for each $n \in \mathbb{N}$ imply that the series $\sum_{m=1}^{\infty} T_{k, m}$ converges for each $k \in \mathbb{N}$. In other words, the rows of the matrix of $T$ are summable. Set

$$
\mathcal{D}\left(\mathfrak{J}_{p}\right):=\left\{T \in \mathcal{B}\left(\mathfrak{J}_{p}\right) \mid \sum_{m=1}^{\infty} T_{k, m}=0 \text { for each } k \in \mathbb{N} \text { and }\left(T_{k, m}\right)_{k, m=1}^{\infty}\right. \text { has only }
$$

finitely many non-zero entries in each row and column $\}$.

Loy and Willis note that an operator $T$ on $\mathfrak{J}_{2}$ is weakly compact if and only if the sequence $\left(\sum_{m=1}^{\infty} T_{k, m}\right)_{k \in \mathbb{N}}$ converges to zero (see [21, p. 328]). This observation is also true for operators on $\mathfrak{J}_{p}$ for any $\left.p \in\right] 1, \infty\left[\right.$. It follows that $\mathcal{D}\left(\mathfrak{J}_{p}\right)$ is contained in $\mathcal{W}\left(\mathfrak{J}_{p}\right)$. Conversely, each weakly compact operator on $\mathfrak{J}_{p}$ is the sum of a compact operator and an operator belonging to $\mathcal{D}\left(\mathfrak{J}_{p}\right)$. This result is proved by Loy and Willis for $p=2$ (see $[\mathbf{2 1}$, Lemma 2.1]). Their proof carries over to the general case provided that the reference to $[\mathbf{1}, 3.2]$ is replaced with the elementary observation that, for each $\left(\alpha_{k}\right)_{k \in \mathbb{N}} \in \mathfrak{J}_{p}$, the coordinatewise multiplication map $\left(\beta_{k}\right)_{k \in \mathbb{N}} \mapsto\left(\alpha_{k} \beta_{k}\right)_{k \in \mathbb{N}}$ defines a compact operator on $\mathfrak{J}_{p}$ of norm at most $2\left\|\left(\alpha_{k}\right)_{k \in \mathbb{N}}\right\|_{\mathfrak{J}_{p}}$. In conclusion, we have

$$
\mathcal{W}\left(\mathfrak{J}_{p}\right)=\mathcal{D}\left(\mathfrak{J}_{p}\right)+\mathcal{K}\left(\mathfrak{J}_{p}\right) .
$$

The left shift

$$
\Lambda: \sum_{k=1}^{\infty} \alpha_{k} e_{k} \mapsto \sum_{k=1}^{\infty} \alpha_{k+1} e_{k}, \quad \mathfrak{J}_{p} \rightarrow \mathfrak{J}_{p}
$$


is an operator of norm one on $\mathfrak{J}_{p}$, and hence

$$
A_{n, \boldsymbol{j}}:=\frac{1}{n} H_{j} \sum_{k=0}^{n-1} \Lambda^{k} \quad(n \in \mathbb{N}, j \in \mathbb{J})
$$

is an operator of norm at most one on $\mathfrak{J}_{p}$. The operators $A_{n, \boldsymbol{j}}$ are at the heart of Loy and Willis's construction of a bounded right approximate identity in $\mathcal{W}\left(\mathfrak{J}_{2}\right)$ (see $[\mathbf{2 1}$, Theorem 2.3]; note that $A_{n, \boldsymbol{j}}$ is denoted by $A(n, \boldsymbol{j})$ in [21]). Loy and Willis's proof can be adapted to work for any $p \in] 1, \infty[$, yielding the following key result.

Lemma 4.13. For each operator $T$ belonging to $\mathcal{D}\left(\mathfrak{J}_{p}\right)$, there is an element $\boldsymbol{j} \in \mathbb{J}$ such that

$$
\left\|T A_{n, \boldsymbol{j}}\right\| \leqslant \sqrt[p^{*}]{\frac{2}{n}}\|T\| \quad(n \in \mathbb{N}),
$$

where $p^{*}=p /(p-1)$ is the conjugate exponent of $p$.

Lemma 4.14. The operator $I_{\mathfrak{J}_{p}}-\Lambda$ belongs to the ideal $\mathcal{P}_{I_{\ell_{p}}}\left(\mathfrak{J}_{p}\right)$.

Proof. This is immediate from the fact that $I_{\mathfrak{J}_{p}}-\Lambda=\Theta \Delta$, where

$$
\Delta:\left(\alpha_{k}\right)_{k \in \mathbb{N}} \mapsto\left(\alpha_{k}-\alpha_{k+1}\right)_{k \in \mathbb{N}}, \quad \mathfrak{J}_{p} \rightarrow \ell_{p},
$$

is the coordinatewise difference operator, and

$$
\Theta:\left(\alpha_{k}\right)_{k \in \mathbb{N}} \mapsto\left(\alpha_{k}\right)_{k \in \mathbb{N}}, \quad \ell_{p} \rightarrow \mathfrak{J}_{p}
$$

is the formal inclusion operator.

Corollary 4.15. For each operator $T$ belonging to $\mathcal{D}\left(\mathfrak{J}_{p}\right)$, there is an element $\boldsymbol{j} \in \mathbb{J}$ such that $T H_{j}$ belongs to $\mathcal{Q}_{I_{\ell_{p}}}\left(\mathfrak{J}_{p}\right)$.

Proof. Let $\pi: \mathcal{B}\left(\mathfrak{J}_{p}\right) \rightarrow \mathcal{B}\left(\mathfrak{J}_{p}\right) / \mathcal{Q}_{\ell_{\ell_{p}}}\left(\mathfrak{J}_{p}\right)$ be the quotient homomorphism, and take $\boldsymbol{j} \in$ $\mathbb{J}$ as in Lemma 4.13. Then, for each $n \in \mathbb{N}$, we have

$$
\sqrt[p^{*}]{\frac{2}{n}}\|T\| \geqslant\left\|T A_{n, \boldsymbol{j}}\right\| \geqslant\left\|\pi\left(T A_{n, \boldsymbol{j}}\right)\right\|=\left\|\pi(T) \frac{1}{n} \pi\left(H_{\boldsymbol{j}}\right) \sum_{k=0}^{n-1} \pi(\Lambda)^{k}\right\|=\left\|\pi\left(T H_{\boldsymbol{j}}\right)\right\|
$$

because $\pi(\Lambda)=\pi\left(I_{\mathfrak{J}_{p}}\right)$ by Lemma 4.14 . Since $\sqrt[p^{*}]{2 / n}\|T\| \rightarrow 0$ as $n \rightarrow \infty$ and $\left\|\pi\left(T H_{j}\right)\right\|$ is independent of $n$, we conclude that $\pi\left(T H_{\boldsymbol{j}}\right)=0$, and the result follows.

Theorem 4.16. The ideal $\mathcal{W}\left(\mathfrak{J}_{p}\right)$ of weakly compact operators is the unique maximal ideal in $\mathcal{B}\left(\mathfrak{J}_{p}\right)$.

Proof. The fact that the ideal $\mathcal{W}\left(\mathfrak{J}_{p}\right)$ has codimension one in $\mathcal{B}\left(\mathfrak{J}_{p}\right)$ implies that it is maximal.

To see that it is unique, we show that each proper, closed ideal $\mathcal{I}$ in $\mathcal{B}\left(\mathfrak{J}_{p}\right)$ is contained in $\mathcal{W}\left(\mathfrak{J}_{p}\right)$. If $\mathcal{I}=\{0\}$ or $\mathcal{I}=\mathcal{K}\left(\mathfrak{J}_{p}\right)$, then this is certainly true. Otherwise Corollary 4.12 implies that $\mathcal{Q}_{I_{\ell_{p}}}\left(\mathfrak{J}_{p}\right)$ is contained in $\mathcal{I}$. Each operator $R$ on $\mathfrak{J}_{p}$ can be written 
$R=\zeta I_{\mathfrak{J}_{p}}+S$, where $\zeta$ belongs to $\mathbb{C}$ and $S$ belongs to $\mathcal{W}\left(\mathfrak{J}_{p}\right)$. By (4.5), we can take $T$ in $\mathcal{D}\left(\mathfrak{J}_{p}\right)$ and $K$ in $\mathcal{K}\left(\mathfrak{J}_{p}\right)$ such that $S=T+K$. Corollary 4.15 implies that $T H_{j}$ belongs to $\mathcal{Q}_{I_{\ell_{p}}}\left(\mathfrak{J}_{p}\right)$ (and hence to $\mathcal{I}$ ) for some $\boldsymbol{j} \in \mathbb{J}$. Suppose that $R$ belongs to $\mathcal{I}$. Then, by (4.2), we obtain

$$
\zeta I_{\mathfrak{J}_{p}}=G_{\boldsymbol{j}}(R-S) H_{\boldsymbol{j}}=G_{\boldsymbol{j}}\left(R H_{\boldsymbol{j}}-T H_{\boldsymbol{j}}-K H_{\boldsymbol{j}}\right) \in \mathcal{I} .
$$

It follows that $\zeta=0$, and consequently $R$ belongs to $\mathcal{W}\left(\mathfrak{J}_{p}\right)$.

A similar result holds for the algebra $\mathcal{B}\left(\mathfrak{J}_{p}^{\oplus n}\right)$ of operators on the direct sum of $n$ copies of $\mathfrak{J}_{p}$, where $n$ is any natural number. This is an immediate consequence of the above theorem, the identifications of $\mathcal{B}\left(\mathfrak{J}_{p}^{\oplus n}\right)$ and $\mathcal{W}\left(\mathfrak{J}_{p}^{\oplus n}\right)$ with $M_{n}\left(\mathcal{B}\left(\mathfrak{J}_{p}\right)\right)$ and $M_{n}\left(\mathcal{W}\left(\mathfrak{J}_{p}\right)\right)$, respectively, and the standard algebraic fact [23, Proposition 1.6.10].

Corollary 4.17. For each natural number $n$, the ideal $\mathcal{W}\left(\mathfrak{J}_{p}^{\oplus n}\right)$ of weakly compact operators is the unique maximal ideal in $\mathcal{B}\left(\mathfrak{J}_{p}^{\oplus n}\right)$.

Finally, Corollary 4.15 enables us to complete the characterization of the ideals $\mathcal{Q}_{P_{j}}\left(\mathfrak{J}_{p}\right)$ for $\boldsymbol{j} \in \mathbb{J}$ begun in Proposition 4.4. Our result shows in particular that $\mathcal{W}\left(\mathfrak{J}_{p}\right)$ is the closed ideal generated by a single idempotent operator.

Proposition 4.18. Let $\boldsymbol{j}=\left(j_{k}\right)_{k \in \mathbb{N}_{0}} \in \mathbb{J}$. If the sequence $\left(j_{k}-j_{k-1}\right)_{k \in \mathbb{N}}$ is unbounded, then

$$
\mathcal{Q}_{P_{j}}\left(\mathfrak{J}_{p}\right)=\mathcal{W}\left(\mathfrak{J}_{p}\right) .
$$

Proof. We already know that $\mathcal{K}\left(\mathfrak{J}_{p}\right) \subseteq \mathcal{Q}_{I_{\ell_{p}}}\left(\mathfrak{J}_{p}\right) \subseteq \mathcal{Q}_{P_{j}}\left(\mathfrak{J}_{p}\right) \subseteq \mathcal{W}\left(\mathfrak{J}_{p}\right)$, so by (4.5) it suffices to show that $\mathcal{D}\left(\mathfrak{J}_{p}\right)$ is contained in $\mathcal{Q}_{P_{j}}\left(\mathfrak{J}_{p}\right)$. For each $T$ in $\mathcal{D}\left(\mathfrak{J}_{p}\right)$, Corollary 4.15 implies that $T H_{\boldsymbol{i}}$ belongs to $\mathcal{Q}_{I_{\ell_{p}}}\left(\mathfrak{J}_{p}\right)$ for some $\boldsymbol{i} \in \mathbb{J}$. It follows from Proposition 4.4 that $\operatorname{im} P_{\boldsymbol{i}}$ is isomorphic to a complemented subspace of im $P_{\boldsymbol{j}}$. Hence $P_{\boldsymbol{i}}$ belongs to $\mathcal{Q}_{P_{\boldsymbol{j}}}\left(\mathfrak{J}_{p}\right)$, and so

$$
T=T P_{i}+T H_{i} G_{i} \in \mathcal{Q}_{P_{j}}\left(\mathfrak{J}_{p}\right),
$$

as required.

Question 4.19. Are there any closed ideals in $\mathcal{B}\left(\mathfrak{J}_{p}\right)$ between $\mathcal{Q}_{I_{\ell_{p}}}\left(\mathfrak{J}_{p}\right)$ and $\mathcal{W}\left(\mathfrak{J}_{p}\right)$ ?

\section{The Brown-McCoy radical of $\mathcal{B}(\mathfrak{X})$}

In this section we calculate the Brown-McCoy radical of the algebra $\mathcal{B}(\mathfrak{X})$ for various Banach spaces $\mathfrak{X}$, and we show that it cannot be turned into an operator ideal.

Definition 5.1. Let $\mathcal{B}$ be a unital algebra. The Brown-McCoy radical (also known as the strong radical) of $\mathcal{B}$ is the intersection of all maximal ideals in $\mathcal{B}$. The Jacobson radical of $\mathcal{B}$ is the intersection of all maximal left ideals in $\mathcal{B}$. We denote these radicals by $\operatorname{rad}_{\mathrm{BM}} \mathcal{B}$ and $\operatorname{rad}_{\mathrm{J}} \mathcal{B}$, respectively.

The Brown-McCoy and Jacobson radicals are proper ideals in $\mathcal{B}$. The Brown-McCoy radical always contains the Jacobson radical by [23, Theorem 4.5.9]. In the case where $\mathcal{B}$ is a unital Banach algebra, maximal (left) ideals are automatically closed, so that 
the Brown-McCoy and Jacobson radicals are closed ideals in $\mathcal{B}$. We refer to $[\mathbf{2 3}, \S \S 4.3$ and 4.5] for further results on these radicals as well as their definitions in the non-unital case.

It is well known that $\operatorname{rad}_{J} \mathcal{B}(\mathfrak{X})=\{0\}$ for each Banach space $\mathfrak{X}$. In contrast to this, we note that

$$
\mathcal{A}(\mathfrak{X}) \subseteq \operatorname{rad}_{\mathrm{BM}} \mathcal{B}(\mathfrak{X})
$$

whenever the Banach space $\mathfrak{X}$ is infinite dimensional. It follows from Theorem 1.1 that

$$
\operatorname{rad}_{\mathrm{BM}} \mathcal{B}\left(c_{0}\right)=\mathcal{K}\left(c_{0}\right)=\mathcal{A}\left(c_{0}\right) \quad \text { and } \quad \operatorname{rad}_{\mathrm{BM}} \mathcal{B}\left(\ell_{p}\right)=\mathcal{K}\left(\ell_{p}\right)=\mathcal{A}\left(\ell_{p}\right) \quad(1 \leqslant p<\infty)
$$

so that the Brown-McCoy radical is as 'small' as possible in these cases. On the other hand, Corollary 4.17 shows that the inclusion in (5.1) may be proper since

$$
\operatorname{rad}_{\mathrm{BM}} \mathcal{B}\left(\mathfrak{J}_{p}^{\oplus n}\right)=\mathcal{W}\left(\mathfrak{J}_{p}^{\oplus n}\right) \supsetneq \mathcal{A}\left(\mathfrak{J}_{p}^{\oplus n}\right) \quad(n \in \mathbb{N}, 1<p<\infty) .
$$

Yood observed that the Jacobson radical of $\mathcal{B}(\mathfrak{X}) / \mathcal{A}(\mathfrak{X})$ is non-zero for certain Banach spaces $\mathfrak{X}$ (see [31, p. 615]). Kleinecke then defined the ideal of inessential operators by

$$
\mathcal{E}(\mathfrak{X})=\pi^{-1}\left(\operatorname{rad}_{\mathrm{J}}(\mathcal{B}(\mathfrak{X}) / \mathcal{A}(\mathfrak{X}))\right)
$$

for each Banach space $\mathfrak{X}$, where $\pi: \mathcal{B}(\mathfrak{X}) \rightarrow \mathcal{B}(\mathfrak{X}) / \mathcal{A}(\mathfrak{X})$ is the quotient homomorphism (see $[\mathbf{1 7}])$. Subsequently, Pietsch gave the 'operator ideal' definition of $\mathcal{E}(\mathfrak{X}, \mathfrak{Y})$ that we used on p. 526, and showed that it coincides with Kleinecke's original definition (5.3) for $\mathfrak{X}=\mathfrak{Y}($ see $[\mathbf{2 4}, 26.7 .2]$ and $[\mathbf{1 7}$, Theorem 1]).

It follows from (5.1) and [23, Theorem 4.5.3(b)] that

$$
\pi^{-1}\left(\operatorname{rad}_{\mathrm{BM}}(\mathcal{B}(\mathfrak{X}) / \mathcal{A}(\mathfrak{X}))\right)=\operatorname{rad}_{\mathrm{BM}} \mathcal{B}(\mathfrak{X})
$$

for each infinite-dimensional Banach space $\mathfrak{X}$. Our aim is to show that-unlike Pietsch's observation that the assignment $\mathfrak{X} \mapsto \pi^{-1}\left(\operatorname{rad}_{\mathrm{J}}(\mathcal{B}(\mathfrak{X}) / \mathcal{A}(\mathfrak{X}))\right)$ can be turned into an operator ideal $\mathcal{E}$-it is impossible to turn the assignment

$$
\mathfrak{X} \mapsto \pi^{-1}\left(\operatorname{rad}_{\mathrm{BM}}(\mathcal{B}(\mathfrak{X}) / \mathcal{A}(\mathfrak{X}))\right)=\operatorname{rad}_{\mathrm{BM}} \mathcal{B}(\mathfrak{X})
$$

into an operator ideal. To make this statement precise, we give the following definition.

Definition 5.2. Let $\mathfrak{X} \mapsto \mathcal{J}_{\mathfrak{X}}$ be an assignment which associates with each infinitedimensional Banach space $\mathfrak{X}$ an ideal $\mathcal{J}_{\mathfrak{X}}$ in $\mathcal{B}(\mathfrak{X})$. If there is an operator ideal $\mathcal{I}$ such that $\mathcal{I}(\mathfrak{X})=\mathcal{J}_{\mathfrak{X}}$ for each infinite-dimensional Banach space $\mathfrak{X}$, then we say that the assignment $\mathfrak{X} \mapsto \mathcal{J}_{\mathfrak{X}}$ can be turned into an operator ideal.

Theorem 5.3. The assignment $\mathfrak{X} \mapsto \operatorname{rad}_{\mathrm{BM}} \mathcal{B}(\mathfrak{X})$ cannot be turned into an operator ideal.

Proof. Assume towards a contradiction that $\mathcal{I}$ is an operator ideal satisfying $\mathcal{I}(\mathfrak{X})=$ $\operatorname{rad}_{\mathrm{BM}} \mathcal{B}(\mathfrak{X})$ for each infinite-dimensional Banach space $\mathfrak{X}$, and take any $\left.p \in\right] 1, \infty[$. It 
follows from Proposition 4.4 (iii) that there are operators $S: \mathfrak{J}_{p} \rightarrow \ell_{p}$ and $T: \ell_{p} \rightarrow \mathfrak{J}_{p}$ such that $S T=I_{\ell_{p}}$. Since

$$
T S \in \mathcal{P}_{I_{\ell_{p}}}\left(\mathfrak{J}_{p}\right) \subseteq \mathcal{W}\left(\mathfrak{J}_{p}\right)=\operatorname{rad}_{\mathrm{BM}} \mathcal{B}\left(\mathfrak{J}_{p}\right)=\mathcal{I}\left(\mathfrak{J}_{p}\right),
$$

we conclude that

$$
I_{\ell_{p}}=S(T S) T \in \mathcal{I}\left(\ell_{p}\right)=\operatorname{rad}_{\mathrm{BM}} \mathcal{B}\left(\ell_{p}\right),
$$

contradicting the fact that $\operatorname{rad}_{\mathrm{BM}} \mathcal{B}\left(\ell_{p}\right)$ is a proper ideal in $\mathcal{B}\left(\ell_{p}\right)$.

\section{Maximal ideal structure of $\mathcal{B}(\mathfrak{X})$}

The aim of this section is to construct, for each natural number $n$, a Banach space $\mathfrak{X}$ such that $\mathcal{B}(\mathfrak{X})$ has exactly $n$ maximal ideals, and each of these $n$ maximal ideals has a prescribed codimension from the set $\left\{k^{2} \mid k \in \mathbb{N}\right\} \cup\{\infty\}$. Note that, by Wedderburn's Theorem (see [23, Theorem 8.1.1]), the square numbers and infinity are the only possible codimensions of maximal ideals in $\mathcal{B}(\mathfrak{X})$, and so this result is the 'best possible'.

We begin with some elementary, but very useful, algebraic observations which extend the work of Volkmann (see [29]). Take an integer $n \geqslant 2$, let $\mathfrak{W}_{1}, \ldots, \mathfrak{W}_{n}$ be vector spaces, and set $\mathfrak{W}:=\mathfrak{W}_{1} \oplus \cdots \oplus \mathfrak{W}_{n}$. Recall from $\S 2$ that $J_{1}, \ldots, J_{n}$ are the canonical coordinate embeddings and $Q_{1}, \ldots, Q_{n}$ are the canonical coordinate projections associated with the direct sum, and consider a subalgebra $\mathcal{B}$ of $\mathcal{L}(\mathfrak{W})$ satisfying

$$
J_{j} Q_{j} \in \mathcal{B} \quad(j \in\{1, \ldots, n\}) .
$$

Observe that $I_{\mathfrak{W}}=\sum_{j=1}^{n} J_{j} Q_{j} \in \mathcal{B}$, so that $\mathcal{B}$ is unital.

For $j, k \in\{1, \ldots, n\}$, set $\mathcal{B}_{j, k}:=Q_{j} \mathcal{B} J_{k}$. This is a linear subspace of $\mathcal{L}\left(\mathfrak{W}_{k}, \mathfrak{W}_{j}\right)$, and for a linear map $T: \mathfrak{W}_{k} \rightarrow \mathfrak{W}_{j}$ we have: $T$ belongs to $\mathcal{B}_{j, k}$ if and only if $J_{j} T Q_{k}$ belongs to $\mathcal{B}$. We write $\mathcal{B}_{j}$ instead of $\mathcal{B}_{j, j}$. This is a unital subalgebra of $\mathcal{L}\left(\mathfrak{W}_{j}\right)$.

\section{Lemma 6.1.}

(i) Let $j \in\{1, \ldots, n\}$, let $\mathcal{I}$ be a subset of $\mathcal{B}_{j}$, and set

$$
\widehat{\mathcal{I}}:=\left\{T \in \mathcal{B} \mid Q_{j} T J_{j} \in \mathcal{I}\right\} .
$$

Then $Q_{j} \widehat{\mathcal{I}} J_{j}=\mathcal{I}$. Moreover, $\mathcal{I}$ is an ideal in $\mathcal{B}_{j}$ satisfying

$$
\mathcal{B}_{j, k} \mathcal{B}_{k, j} \subseteq \mathcal{I} \quad(k \in\{1, \ldots, n\} \backslash\{j\})
$$

if and only if $\widehat{\mathcal{I}}$ is an ideal in $\mathcal{B}$.

(ii) Let $\mathcal{I}$ be an ideal in $\mathcal{B}$, and let $j \in\{1, \ldots, n\}$. Then

$$
Q_{j} \mathcal{I} J_{j}=\left\{T \in \mathcal{B}_{j} \mid J_{j} T Q_{j} \in \mathcal{I}\right\},
$$

and this is an ideal in $\mathcal{B}_{j}$. 
In the following we write $\mathcal{I} \unlhd \mathcal{A}$ to denote that $\mathcal{I}$ is an ideal in an algebra $\mathcal{A}$. For each $j \in\{1, \ldots, n\}$, the set

$$
\mathbb{I}_{j}:=\left\{\mathcal{I} \unlhd \mathcal{B}_{j} \mid \mathcal{B}_{j, k} \mathcal{B}_{k, j} \subseteq \mathcal{I}(k \in\{1, \ldots, n\} \backslash\{j\})\right\}
$$

of ideals in $\mathcal{B}_{j}$ satisfying (6.2) is a sublattice of the lattice of ideals in $\mathcal{B}_{j}$, and the set

$$
\mathbb{I}:=\left\{\mathcal{I} \unlhd \mathcal{B} \mid J_{j} \mathcal{B}_{j, k} Q_{k} \subseteq \mathcal{I}(j, k \in\{1, \ldots, n\}, j \neq k)\right\}
$$

is a sublattice of the lattice of ideals in $\mathcal{B}$. These lattices are related as follows.

Lemma 6.2. Equip $\mathbb{I}_{1} \times \cdots \times \mathbb{I}_{n}$ with the product order. Then the map

$$
\Upsilon: \quad\left(\mathcal{I}_{1}, \ldots, \mathcal{I}_{n}\right) \mapsto \bigcap_{j=1}^{n} \widehat{\mathcal{I}}_{j}, \quad \mathbb{I}_{1} \times \cdots \times \mathbb{I}_{n} \rightarrow \mathbb{I},
$$

where $\widehat{\mathcal{I}}_{j}$ is given by (6.1), is an order isomorphism with inverse

$$
\Upsilon^{-1}: \mathcal{I} \mapsto\left(Q_{1} \mathcal{I} J_{1}, \ldots, Q_{n} \mathcal{I} J_{n}\right), \quad \mathbb{I} \rightarrow \mathbb{I}_{1} \times \cdots \times \mathbb{I}_{n} .
$$

\section{Corollary 6.3.}

(i) Let $j \in\{1, \ldots, n\}$, and let $\mathcal{M}$ be a maximal ideal in $\mathcal{B}_{j}$ containing $\mathcal{B}_{j, k} \mathcal{B}_{k, j}$ for each $k \in\{1, \ldots, n\} \backslash\{j\}$. Then

$$
\Upsilon\left(\mathcal{B}_{1}, \ldots, \mathcal{B}_{j-1}, \mathcal{M}, \mathcal{B}_{j+1}, \ldots, \mathcal{B}_{n}\right)=\left\{T \in \mathcal{B} \mid Q_{j} T J_{j} \in \mathcal{M}\right\}
$$

is a maximal ideal in $\mathcal{B}$.

(ii) Let $\mathcal{N}$ be a maximal ideal in $\mathcal{B}$ containing $J_{j} \mathcal{B}_{j, k} Q_{k}$ whenever $j, k \in\{1, \ldots, n\}$ are distinct. Then $\Upsilon^{-1}(\mathcal{N})=\left(\mathcal{B}_{1}, \ldots, \mathcal{B}_{j-1}, \mathcal{M}, \mathcal{B}_{j+1}, \ldots, \mathcal{B}_{n}\right)$ for some $j \in\{1, \ldots, n\}$ and some maximal ideal $\mathcal{M}$ in $\mathcal{B}_{j}$.

We shall now specialize to the case where $\mathfrak{W}_{1}, \ldots, \mathfrak{W}_{n}$ (and hence $\mathfrak{W}$ ) are Banach spaces and $\mathcal{B}=\mathcal{B}(\mathfrak{W})$. Then $\mathcal{B}_{j, k}=\mathcal{B}\left(\mathfrak{W}_{k}, \mathfrak{W}_{j}\right)$ for each pair $(j, k) \in\{1, \ldots, n\}^{2}$.

We shall restrict our attention to closed ideals. The lattice isomorphism $\Upsilon$ preserves closed ideals in the strong sense that, for $\left(\mathcal{I}_{1}, \ldots, \mathcal{I}_{n}\right) \in \mathbb{I}_{1} \times \cdots \times \mathbb{I}_{n}$, we have that $\mathcal{I}_{1}, \ldots, \mathcal{I}_{n}$ are closed if and only if $\Upsilon\left(\mathcal{I}_{1}, \ldots, \mathcal{I}_{n}\right)$ is closed. It follows that we may regard $\Upsilon$ as a lattice isomorphism from $\mathbb{I}_{1}^{\mathrm{cl}} \times \cdots \times \mathbb{I}_{n}^{\mathrm{cl}}$ onto $\mathbb{I}^{\mathrm{cl}}$, where $\mathbb{I}_{1}^{\mathrm{cl}}, \ldots, \mathbb{I}_{n}^{\mathrm{cl}}$ and $\mathbb{I}^{\mathrm{cl}}$ are the lattices consisting of the closed ideals belonging to $\mathbb{I}_{1}, \ldots, \mathbb{I}_{n}$ and $\mathbb{I}$, respectively.

We shall further assume that there are only a 'few' operators between any two spaces $\mathfrak{W}_{j}$ and $\mathfrak{W}_{k}$ whenever $j, k \in\{1, \ldots, n\}$ are distinct. To make this precise, we require González's concept of essentially incomparable Banach spaces defined in the following proposition.

Proposition 6.4 (González [9]). Let $\mathfrak{X}$ and $\mathfrak{Y}$ be Banach spaces. Each operator from $\mathfrak{X}$ to $\mathfrak{Y}$ is inessential if and only if each operator from $\mathfrak{Y}$ to $\mathfrak{X}$ is inessential.

If either (and hence both) of these assertions is satisfied, then $\mathfrak{X}$ and $\mathfrak{Y}$ are termed essentially incomparable. 
Lemma 6.5. Let $\mathfrak{W}_{1}, \ldots, \mathfrak{W}_{n}$ be pairwise essentially incomparable Banach spaces. For each $j \in\{1, \ldots, n\}$, the set

$$
\mathbb{E}_{j}:=\left\{\mathcal{I} \unlhd \mathcal{B}\left(\mathfrak{W}_{j}\right) \mid \mathcal{I} \text { is closed and } \mathcal{E}\left(\mathfrak{W}_{j}\right) \subseteq \mathcal{I}\right\}
$$

is a sublattice of $\mathbb{I}_{j}^{\mathrm{cl}}$, and $\Upsilon$ maps $\mathbb{E}_{1} \times \cdots \times \mathbb{E}_{n}$ onto the sublattice

$$
\mathbb{E}:=\{\mathcal{I} \unlhd \mathcal{B}(\mathfrak{W}) \mid \mathcal{I} \text { is closed and } \mathcal{E}(\mathfrak{W}) \subseteq \mathcal{I}\}
$$

of $\mathbb{I}^{\mathrm{cl}}$.

Proof. Let $j, k \in\{1, \ldots, n\}$ be distinct. Then $\mathcal{B}_{j, k}=\mathcal{E}\left(\mathfrak{W}_{k}, \mathfrak{W}_{j}\right)$ because $\mathfrak{W}_{j}$ and $\mathfrak{W}_{k}$ are essentially incomparable. It follows that $\mathcal{B}_{j, k} \mathcal{B}_{k, j} \subseteq \mathcal{E}\left(\mathfrak{W}_{j}\right)$ and $J_{j} \mathcal{B}_{j, k} Q_{k} \subseteq \mathcal{E}(\mathfrak{W})$, and consequently $\mathbb{E}_{j}$ and $\mathbb{E}$ are subsets of $\mathbb{I}_{j}^{\mathrm{cl}}$ and $\mathbb{I}^{\mathrm{cl}}$, respectively. It is clear that $\mathbb{E}_{j}$ and $\mathbb{E}$ are closed under the lattice operations.

If $\left(\mathcal{I}_{1}, \ldots, \mathcal{I}_{n}\right) \in \mathbb{E}_{1} \times \cdots \times \mathbb{E}_{n}$, then $Q_{j} \mathcal{E}(\mathfrak{W}) J_{j}=\mathcal{E}\left(\mathfrak{W}_{j}\right) \subseteq \mathcal{I}_{j}$ for each $j \in\{1, \ldots, n\}$, and hence $\mathcal{E}(\mathfrak{W}) \subseteq \bigcap_{j=1}^{n} \widehat{\mathcal{I}}_{j}=\Upsilon\left(\mathcal{I}_{1}, \ldots, \mathcal{I}_{n}\right)$. This shows that $\Upsilon\left(\mathbb{E}_{1} \times \cdots \times \mathbb{E}_{n}\right) \subseteq \mathbb{E}$.

Conversely, if $\mathcal{I} \in \mathbb{E}$, then $\mathcal{E}\left(\mathfrak{W}_{j}\right)=Q_{j} \mathcal{E}(\mathfrak{W}) J_{j} \subseteq Q_{j} \mathcal{I} J_{j}$ for each $j \in\{1, \ldots, n\}$, so that $\Upsilon^{-1}(\mathcal{I}) \in \mathbb{E}_{1} \times \cdots \times \mathbb{E}_{n}$.

The importance of the above lemma lies in the fact that we can improve the identity (5.1) to yield that, for each infinite-dimensional Banach space $\mathfrak{X}$, the ideal of inessential operators on $\mathfrak{X}$ is contained in the Brown-McCoy radical of $\mathcal{B}(\mathfrak{X})$, so that maximal ideals automatically belong to the lattices $\mathbb{E}_{j}$ and $\mathbb{E}$.

Proposition 6.6. Let $\mathfrak{X}$ be an infinite-dimensional Banach space. Then

$$
\mathcal{E}(\mathfrak{X}) \subseteq \operatorname{rad}_{\mathrm{BM}} \mathcal{B}(\mathfrak{X}) .
$$

Proof. By $\left[\mathbf{2 3}\right.$, Theorem 4.5.9], $\operatorname{rad}_{\mathrm{J}}(\mathcal{B}(\mathfrak{X}) / \mathcal{A}(\mathfrak{X})) \subseteq \operatorname{rad}_{\mathrm{BM}}(\mathcal{B}(\mathfrak{X}) / \mathcal{A}(\mathfrak{X}))$, and hence the result follows from (5.3) and (5.4).

Remark 6.7. The identity (5.2) gives explicit examples of Banach spaces $\mathfrak{X}$ for which the inclusion $\mathcal{E}(\mathfrak{X}) \subseteq \operatorname{rad}_{\mathrm{BM}} \mathcal{B}(\mathfrak{X})$ is proper.

Corollary 6.8. Let $\mathfrak{W}_{1}, \ldots, \mathfrak{W}_{n}$ be pairwise essentially incomparable, infinite-dimensional Banach spaces. Then the map

$$
\begin{aligned}
(j, \mathcal{M}) & \mapsto\left\{T \in \mathcal{B}(\mathfrak{W}) \mid Q_{j} T J_{j} \in \mathcal{M}\right\} \\
& \bigcup_{j=1}^{n}\left\{(j, \mathcal{M}) \mid \mathcal{M} \text { maximal ideal in } \mathcal{B}\left(\mathfrak{W}_{j}\right)\right\} \rightarrow\{\mathcal{N} \mid \mathcal{N} \text { maximal ideal in } \mathcal{B}(\mathfrak{W})\}
\end{aligned}
$$

is a bijection.

We note in particular the following special case, which improves [29, Satz 2(c)]. 
Corollary 6.9. Let $\mathfrak{W}_{1}, \ldots, \mathfrak{W}_{n}$ be pairwise essentially incomparable, infinite-dimensional Banach spaces. For each $j \in\{1, \ldots, n\}$, let $\mathcal{M}_{j}$ be a subset of $\mathcal{B}\left(\mathfrak{W}_{j}\right)$, and set

$$
\mathcal{N}_{j}:=\left\{T \in \mathcal{B}(\mathfrak{W}) \mid Q_{j} T J_{j} \in \mathcal{M}_{j}\right\} .
$$

Then $\mathcal{M}_{j}$ is the only maximal ideal in $\mathcal{B}\left(\mathfrak{W}_{j}\right)$ for each $j \in\{1, \ldots, n\}$ if and only if $\mathcal{N}_{1}, \ldots, \mathcal{N}_{n}$ are the only maximal ideals in $\mathcal{B}(\mathfrak{W})$.

Remark 6.10. The condition that the Banach spaces $\mathfrak{W}_{1}, \ldots, \mathfrak{W}_{n}$ are pairwise essentially incomparable cannot be removed from the above results. For example, take $\mathfrak{W}_{1}=\ell_{p}$ and $\mathfrak{W}_{2}=\mathfrak{J}_{p}$ for some $\left.p \in\right] 1, \infty[$. These spaces are not essentially incomparable. We know that $\mathcal{K}\left(\mathfrak{W}_{1}\right)$ is the unique maximal ideal in $\mathcal{B}\left(\mathfrak{W}_{1}\right)$ and that $\mathcal{W}\left(\mathfrak{W}_{2}\right)$ is the unique maximal ideal in $\mathcal{B}\left(\mathfrak{W}_{2}\right)$. However, $\mathcal{B}\left(\mathfrak{W}_{1} \oplus \mathfrak{W}_{2}\right)$ also has a unique maximal ideal because $\mathfrak{W}_{1} \oplus \mathfrak{W}_{2}$ is isomorphic to $\mathfrak{W}_{2}$ by Lemma 4.2 and Proposition 4.4 (iii). This shows that Corollary 6.9 fails in this case.

The following fact is a consequence of Remark 3.2; it extends [9, Observation 1(b)].

Lemma 6.11. Let $m$ and $n$ be natural numbers, and let $\mathfrak{X}_{1}, \ldots, \mathfrak{X}_{n}$ and $\mathfrak{Y}_{1}, \ldots, \mathfrak{Y}_{m}$ be Banach spaces. Then $\mathfrak{X}_{1} \oplus \cdots \oplus \mathfrak{X}_{n}$ and $\mathfrak{Y}_{1} \oplus \cdots \oplus \mathfrak{Y}_{m}$ are essentially incomparable if and only if $\mathfrak{X}_{k}$ and $\mathfrak{Y}_{j}$ are essentially incomparable for each pair $(j, k) \in\{1, \ldots, m\} \times$ $\{1, \ldots, n\}$.

We are now ready to prove our main result; it classifies all the closed ideals in $\mathcal{B}(\mathfrak{X})$ containing $\mathcal{E}(\mathfrak{X})$, where $\mathfrak{X}$ is a finite direct sum of James spaces and $\ell_{p}$-spaces. In particular it shows that for any choice of finitely many square numbers, there is a Banach space $\mathfrak{X}$ such that $\mathcal{B}(\mathfrak{X})$ has maximal ideals of these codimensions; and in addition there may be any finite number of maximal ideals of infinite codimension.

Theorem 6.12. Let $n, m$ and $\nu_{1}, \ldots, \nu_{n}$ be natural numbers, let $p_{1}, \ldots, p_{n+m}$ be distinct real numbers with $\left.p_{1}, \ldots, p_{n} \in\right] 1, \infty\left[\right.$ and $p_{n+1}, \ldots, p_{n+m} \in[1, \infty[$, and set

$$
\mathfrak{X}:=\mathfrak{J}_{p_{1}}^{\oplus \nu_{1}} \oplus \mathfrak{J}_{p_{2}}^{\oplus \nu_{2}} \oplus \cdots \oplus \mathfrak{J}_{p_{n}}^{\oplus \nu_{n}} \oplus \ell_{p_{n+1}} \oplus \ell_{p_{n+2}} \oplus \cdots \oplus \ell_{p_{n+m}} .
$$

Then

$$
\mathbb{E}_{j}= \begin{cases}\left\{\mathcal{I} \unlhd \mathcal{B}\left(\mathfrak{J}_{p_{j}}^{\oplus \nu_{j}}\right) \mid \mathcal{I} \text { is non-zero and closed }\right\}, & 1 \leqslant j \leqslant n, \\ \left\{\mathcal{K}\left(\ell_{p_{j}}\right), \mathcal{B}\left(\ell_{p_{j}}\right)\right\}, & n+1 \leqslant j \leqslant n+m,\end{cases}
$$

and $\Upsilon$ is a lattice isomorphism from $\mathbb{E}_{1} \times \cdots \times \mathbb{E}_{n+m}$ onto

$$
\mathbb{E}=\{\mathcal{I} \unlhd \mathcal{B}(\mathfrak{X}) \mid \mathcal{I} \text { is closed and } \mathcal{E}(\mathfrak{X}) \subseteq \mathcal{I}\} .
$$

Proof. By [21, Theorem 4.5], the Banach spaces $\mathfrak{J}_{p_{1}}, \ldots, \mathfrak{J}_{p_{n}}, \ell_{p_{n+1}}, \ldots, \ell_{p_{n+m}}$ are pairwise essentially incomparable. Lemma 6.11 then implies that

$$
\mathfrak{J}_{p_{1}}^{\oplus \nu_{1}}, \ldots, \mathfrak{J}_{p_{n}}^{\oplus \nu_{n}}, \ell_{p_{n+1}}, \ldots, \ell_{p_{n+m}}
$$

are pairwise essentially incomparable. 
For $j \in\{1, \ldots, n\}, \mathcal{A}\left(\mathfrak{J}_{p_{j}}\right)=\mathcal{E}\left(\mathfrak{J}_{p_{j}}\right)$ by Proposition 4.9, and hence $\mathcal{A}\left(\mathfrak{J}_{p_{j}}^{\oplus \nu_{j}}\right)=\mathcal{E}\left(\mathfrak{J}_{p_{j}}^{\oplus \nu_{j}}\right)$ by Remark 3.2. It follows that $\mathcal{E}\left(\mathfrak{J}_{p_{j}}^{\oplus \nu_{j}}\right)$ is contained in every closed, non-zero ideal in $\mathcal{B}\left(\mathfrak{J}_{p_{j}}^{\oplus \nu_{j}}\right)$. Theorem 1.1 shows that $\mathcal{K}\left(\ell_{p_{j}}\right)=\mathcal{E}\left(\ell_{p_{j}}\right)$ and that $\mathcal{K}\left(\ell_{p_{j}}\right)$ and $\mathcal{B}\left(\ell_{p_{j}}\right)$ are the only closed, non-zero ideals in $\mathcal{B}\left(\ell_{p_{j}}\right)$ for $j \in\{n+1, \ldots, n+m\}$. This proves (6.3). Now the result follows from Lemma 6.5.

Corollary 6.13. Define the Banach space $\mathfrak{X}$ as in Theorem 6.12. Then there are exactly $n+m$ distinct maximal ideals $\mathcal{N}_{1}, \ldots, \mathcal{N}_{n+m}$ in the algebra $\mathcal{B}(\mathfrak{X})$, and they are given by

$$
\mathcal{N}_{j}= \begin{cases}\left\{T \in \mathcal{B}(\mathfrak{X}) \mid Q_{j} T J_{j} \in \mathcal{W}\left(\mathfrak{J}_{p_{j}}^{\oplus \nu_{j}}\right)\right\}, & 1 \leqslant j \leqslant n, \\ \left\{T \in \mathcal{B}(\mathfrak{X}) \mid Q_{j} T J_{j} \in \mathcal{K}\left(\ell_{p_{j}}\right)\right\}, & n+1 \leqslant j \leqslant n+m .\end{cases}
$$

In particular $\mathcal{N}_{j}$ has codimension $\nu_{j}^{2}$ in $\mathcal{B}(\mathfrak{X})$ for $1 \leqslant j \leqslant n$, whereas $\mathcal{N}_{j}$ has infinite codimension in $\mathcal{B}(\mathfrak{X})$ for $n+1 \leqslant j \leqslant n+m$.

\section{Remark 6.14.}

(i) The conclusions of Theorem 6.12 and Corollary 6.13 remain true if we replace one of the $\ell_{p}$-spaces $\ell_{p_{n+1}}, \ldots, \ell_{p_{n+m}}$ by $c_{0}$.

(ii) Special cases of Theorem 6.12 and Corollary 6.13 are obtained by taking either $m=0$ or $n=0$, that is, leaving out either the $\ell_{p}$-spaces $\ell_{p_{n+1}}, \ldots, \ell_{p_{n+m}}$ or the James spaces $\mathfrak{J}_{p_{1}}^{\oplus \nu_{1}}, \ldots, \mathfrak{J}_{p_{n}}^{\oplus \nu_{n}}$. The latter of these cases is the case considered by Volkmann in $[\mathbf{2 9}]$.

\section{Uniqueness of the maximal ideal in $\mathcal{B}(\mathfrak{X})$ for hereditarily indecomposable Banach spaces $\mathfrak{X}$}

In this section we give another example of a class of Banach spaces $\mathfrak{X}$ such that $\mathcal{B}(\mathfrak{X})$ has a unique maximal ideal, and this maximal ideal has finite codimension.

Definition 7.1. An infinite-dimensional Banach space $\mathfrak{X}$ is hereditarily indecomposable if no closed subspace $\mathfrak{W}$ of $\mathfrak{X}$ admits an idempotent operator $P: \mathfrak{W} \rightarrow \mathfrak{W}$ such that neither $\operatorname{im} P$ nor $\operatorname{ker} P$ is finite dimensional.

Gowers and Maurey's fundamental results about hereditarily indecomposable Banach spaces are that they exist and that

$$
\mathcal{B}(\mathfrak{X})=\mathcal{S}(\mathfrak{X})+\mathbb{C} I_{\mathfrak{X}}
$$

for each hereditarily indecomposable Banach space $\mathfrak{X}$ (see $[\mathbf{1 1}]$ ).

Proposition 7.2. Let $\mathfrak{X}$ be a hereditarily indecomposable Banach space. Then $\mathcal{S}(\mathfrak{X})$ is the unique maximal ideal in $\mathcal{B}(\mathfrak{X})$.

More generally, for each natural number $n, \mathcal{S}\left(\mathfrak{X}^{\oplus n}\right)$ is the unique maximal ideal in $\mathcal{B}\left(\mathfrak{X}^{\oplus n}\right)$, and $\operatorname{rad}_{\mathrm{BM}} \mathcal{B}\left(\mathfrak{X}^{\oplus n}\right)=\mathcal{S}\left(\mathfrak{X}^{\oplus n}\right)$. 
Proof. The ideal $\mathcal{S}(\mathfrak{X})$ is maximal by (7.1). Proposition 6.6 implies that each maximal ideal in $\mathcal{B}(\mathfrak{X})$ contains $\mathcal{E}(\mathfrak{X})$ and hence $\mathcal{S}(\mathfrak{X})$. Now the first clause is immediate. The second clause is proved analogously to Corollary 4.17 .

\section{Remark 7.3.}

(i) A completely different class of Banach spaces $\mathfrak{X}$ with the property that the ideal of strictly singular operators is the unique maximal ideal in $\mathcal{B}(\mathfrak{X})$ is described in $[\mathbf{3 0}$, Theorem 6.2$]$, where Whitley proves that this is true for any Banach space $\mathfrak{X}$ such that each closed, infinite-dimensional subspace of $\mathfrak{X}$ contains a subspace which is isomorphic to $\mathfrak{X}$ and complemented in $\mathfrak{X}$. Schlumprecht's space is an interesting example of a Banach space satisfying this condition (see [27]).

(ii) A result similar to Corollary 6.13 can be obtained by replacing the James spaces $\mathfrak{J}_{p_{1}}, \ldots, \mathfrak{J}_{p_{n}}$ by pairwise essentially incomparable, hereditarily indecomposable Banach spaces $\mathfrak{X}_{1}, \ldots, \mathfrak{X}_{n}$ and then applying Proposition 7.2 instead of Corollary 4.17 .

To find such spaces, take any hereditarily indecomposable Banach space $\mathfrak{X}_{1}$ and choose a descending chain $\mathfrak{X}_{1} \supseteq \mathfrak{X}_{2} \supseteq \cdots \supseteq \mathfrak{X}_{n}$ of closed, infinitedimensional subspaces of $\mathfrak{X}_{1}$ such that $\mathfrak{X}_{j+1}$ has infinite codimension in $\mathfrak{X}_{j}$ for each $j \in\{1, \ldots, n-1\}$. It is shown in the proof of Proposition 4.5 in [19] that $\mathcal{B}\left(\mathfrak{X}_{j}, \mathfrak{X}_{k}\right)=\mathcal{S}\left(\mathfrak{X}_{j}, \mathfrak{X}_{k}\right)$ for $1 \leqslant j<k \leqslant n$, so that the spaces $\mathfrak{X}_{1}, \ldots, \mathfrak{X}_{n}$ are pairwise essentially incomparable. It follows from [19, Example 5.1] that, for distinct numbers $p_{1}, \ldots, p_{m} \in\left[1, \infty\left[\right.\right.$, the Banach spaces $\mathfrak{X}_{1}, \ldots, \mathfrak{X}_{n}, \ell_{p_{1}}, \ldots, \ell_{p_{m}}$ are pairwise essentially incomparable.

\section{Classification of the maximal ideals in $\mathcal{B}(\mathfrak{G})$ for the Gowers space $\mathfrak{G}$}

In this section we shall display a Banach space $\mathfrak{G}$ such that the Banach algebra $\mathcal{B}(\mathfrak{G})$ has infinitely many maximal ideals; specifically, there are $2^{2^{\aleph_{0}}}$ distinct maximal ideals in $\mathcal{B}(\mathfrak{G})$, each of codimension one. The Banach space $\mathfrak{G}$ required for this is a creation of Gowers, originally designed to solve Banach's hyperplane problem (see [10]). It has subsequently been investigated in more detail by Gowers and Maurey in [12, Section (5.1)]. Their main result about this space will be stated in Theorem 8.1, below. To do so, we require some elementary facts about certain operators on Banach spaces with an unconditional basis.

Let $\mathfrak{X}$ be a Banach space with an unconditional basis $\boldsymbol{b}=\left(b_{n}\right)_{n \in \mathbb{N}}$. Denote by $\left(g_{n}\right)_{n \in \mathbb{N}}$ the associated biorthogonal functionals. The unconditionality of the basis $\boldsymbol{b}$ means that, for each $\xi=\left(\xi_{n}\right)_{n \in \mathbb{N}} \in \ell_{\infty}$, there is an operator

$$
\Delta_{\boldsymbol{b}}(\xi): x \mapsto \sum_{n=1}^{\infty} \xi_{n} g_{n}(x) b_{n}, \quad \mathfrak{X} \rightarrow \mathfrak{X},
$$

and the norms of these operators are uniformly bounded:

$$
K_{\boldsymbol{b}}:=\sup \left\{\left\|\Delta_{\boldsymbol{b}}(\xi)\right\| \mid \xi \in \ell_{\infty},\|\xi\| \leqslant 1\right\}<\infty .
$$


The assignment $\Delta_{b}: \xi \mapsto \Delta_{b}(\xi), \ell_{\infty} \rightarrow \mathcal{B}(\mathfrak{X})$, is clearly linear and multiplicative, so that $\Delta_{\boldsymbol{b}}$ is a continuous algebra homomorphism. Moreover, $\Delta_{\boldsymbol{b}}$ has a left inverse:

$$
\Gamma_{\boldsymbol{b}}: T \mapsto\left(g_{n}\left(T b_{n}\right)\right)_{n \in \mathbb{N}}, \quad \mathcal{B}(\mathfrak{X}) \rightarrow \ell_{\infty} .
$$

This is an operator of norm at most $K_{\boldsymbol{b}}$; it is not multiplicative. The diagonal operator $\operatorname{diag}_{\boldsymbol{b}}: \mathcal{B}(\mathfrak{X}) \rightarrow \mathcal{B}(\mathfrak{X})$ associated with the unconditional basis $\boldsymbol{b}$ is now defined by

$$
\operatorname{diag}_{\boldsymbol{b}}(T):=\left(\Delta_{\boldsymbol{b}} \Gamma_{\boldsymbol{b}}\right)(T) \quad(T \in \mathcal{B}(\mathfrak{X})) .
$$

Theorem 8.1 (Gowers [10]; Gowers and Maurey [12]). There is a Banach space $\mathfrak{G}$ with an unconditional basis $\boldsymbol{b}$ such that $T-\operatorname{diag}_{\boldsymbol{b}}(T)$ is strictly singular for each $T \in \mathcal{B}(\mathfrak{G})$.

We shall refer to this Banach space $\mathfrak{G}$ as the Gowers space. The basis $\boldsymbol{b}$ will be termed the canonical unconditional basis of $\mathfrak{G}$. Our analysis of $\mathcal{B}(\mathfrak{G})$ relies on the following elementary lemma.

Lemma 8.2. Let $\mathfrak{X}$ be a Banach space with an unconditional basis $\boldsymbol{b}$. For each $\xi \in \ell_{\infty}$, the following assertions are equivalent.

(a) $\xi \in c_{0}$.

(b) $\Delta_{b}(\xi) \in \mathcal{A}(\mathfrak{X})$.

(c) $\Delta_{\boldsymbol{b}}(\xi) \in \mathcal{E}(\mathfrak{X})$

Corollary 8.3. Let $\boldsymbol{b}$ be the canonical unconditional basis of the Gowers space $\mathfrak{G}$, and let $\Pi_{1}: \mathcal{B}(\mathfrak{G}) \rightarrow \mathcal{B}(\mathfrak{G}) / \mathcal{S}(\mathfrak{G})$ and $\Pi_{2}: \ell_{\infty} \rightarrow \ell_{\infty} / c_{0}$ be the quotient homomorphisms. There is a continuous algebra isomorphism $\Psi: \ell_{\infty} / c_{0} \rightarrow \mathcal{B}(\mathfrak{G}) / \mathcal{S}(\mathfrak{G})$ such that the diagram

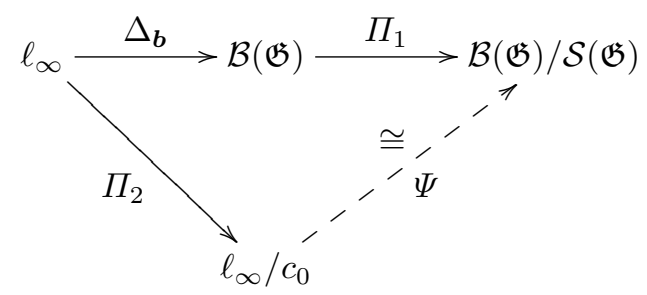

is commutative.

In order to state the main result of this section, we require some terminology. A character on an algebra $\mathcal{A}$ is a non-zero algebra homomorphism from $\mathcal{A}$ to $\mathbb{C}$. For each $m \in \mathbb{N}$, the map $\left(\xi_{n}\right)_{n \in \mathbb{N}} \mapsto \xi_{m}, \ell_{\infty} \rightarrow \mathbb{C}$, is a character on the algebra $\ell_{\infty}$. These characters are termed the point evaluations.

Theorem 8.4. Let $\mathfrak{G}$ be the Gowers space. Each maximal ideal in $\mathcal{B}(\mathfrak{G})$ has codimension one, and the following five sets are in a bijective correspondence to each other:

(a) the set of maximal ideals in $\mathcal{B}(\mathfrak{G})$;

(b) the set of maximal ideals in $\ell_{\infty} / c_{0}$; 
(c) the set of characters on $\ell_{\infty}$ that are not point evaluations;

(d) the set of free ultrafilters on $\mathbb{N}$; and

(e) the set $(\beta \mathbb{N}) \backslash \mathbb{N}$, where $\beta \mathbb{N}$ is the Stone-Čech compactification of $\mathbb{N}$.

In particular there are $2^{2^{\aleph_{0}}}$ distinct maximal ideals in $\mathcal{B}(\mathfrak{G})$.

Proof. Each maximal ideal in $\mathcal{B}(\mathfrak{G})$ contains $\mathcal{S}(\mathfrak{G})$ by Proposition 6.6, so the first clause and the bijective correspondence between the sets in (a) and (b) follow from Corollary 8.3. The bijective correspondence between the sets in (b) and (c) is well known and easy to check, whereas the bijective correspondence between the sets in (c), (d) and (e) is standard; we refer to [7, Chapters $6-7]$ for details. The final clause follows from [7, Theorem 9.2].

Acknowledgements. This work was supported by the European Commission under Marie Curie Research Grant no. ERBFMBI-CT98-3447. I am grateful to H. G. Dales for drawing my attention to two of the main questions addressed in this note, namely: is the character on $\mathcal{B}\left(\mathfrak{J}_{2}\right)$ unique? What is the Brown-McCoy radical of $\mathcal{B}(\mathfrak{X})$ ? I also thank him for his encouragement and helpful suggestions while I carried out this research.

Moreover, I should like to thank H. Jarchow and H.-O. Tylli for several useful comments after I presented a preliminary version of this material at the 14th International Banach Algebras Conference in Claremont, CA.

\section{References}

1. A. D. Andrew and W. L. Green, On James' quasi-reflexive space as a Banach algebra, Can. J. Math. 32 (1980), 1080-1101.

2. E. Berkson and H. Porta, Representations of $\mathfrak{B}(X)$, J. Funct. Analysis 3 (1969), $1-34$.

3. J. W. CALKIN, Two-sided ideals and congruences in the ring of bounded operators in Hilbert space, Ann. Math. 42 (1941), 839-873.

4. S. R. Caradus, W. E. Pfaffennerger, and B. Yood, Calkin algebras and algebras of operators on Banach spaces (Marcel Dekker, 1974).

5. P. G. Casazza, Bor-Luh Lin, and R. H. Lohman, On James' quasi-reflexive Banach space, Proc. Am. Math. Soc. 67 (1977), 265-271.

6. I. S. Edelstein and B. S. Mityagin, Homotopy type of linear groups of two classes of Banach spaces, Funct. Analysis Applic. 4 (1970), 221-231.

7. L. Gillman and M. Jerison, Rings of continuous functions (Van Nostrand Reinhold, New York, 1960).

8. I. C. Gohberg, A. S. Markus, and I. A. Feldman, Normally solvable operators and ideals associated with them, Am. Math. Soc. Transl. 261 (1967), 63-84 (Russian original in Bul. Akad. Stiince RSS Moldoven 10 (1960), 51-70).

9. M. GonzÁLEZ, On essentially incomparable Banach spaces, Math. Z. 215 (1994), 621629 .

10. W. T. Gowers, A solution to Banach's hyperplane problem, Bull. Lond. Math. Soc. 26 (1994), 523-530.

11. W. T. Gowers and B. Maurey, The unconditional basic sequence problem, J. Am. Math. Soc. 6 (1993), 851-874. 
12. W. T. Gowers And B. Maurey, Banach spaces with small spaces of operators, Math. Annln 307 (1997), 543-568.

13. B. Gramsch, Eine Idealstruktur Banachscher Operatoralgebren, J. Reine Angew. Math. 225 (1967), 97-115.

14. P. HABAlA, P. HÁJeK, AND V. Zizler, Introduction to Banach spaces, vol. II (Matfyzpress, Prague, 1996).

15. R. Herman And R. Whitley, An example concerning reflexivity, Studia Math. 28 (1967), 289-294.

16. R. C. JAmes, Bases and reflexivity of Banach spaces, Ann. Math. 52 (1950), 518-527.

17. D. Kleinecke, Almost-finite, compact, and inessential operators, Proc. Am. Math. Soc. 14 (1963), 863-868.

18. E. LACEy AND R. J. Whitley, Conditions under which all the bounded linear maps are compact, Math. Annln 158 (1965), 1-5.

19. N. J. Laustsen, K-theory for algebras of operators on Banach spaces, J. Lond. Math. Soc. 59 (1999), 715-728.

20. J. Lindenstrauss And L. TZAFriri, Classical Banach spaces, vol. 1, Ergebnisse der Mathematik und ihrer Grenzgebiete, vol. 92 (Springer, 1977).

21. R. J. Loy And G. A. Willis, Continuity of derivations on $\mathcal{B}(E)$ for certain Banach spaces E, J. Lond. Math. Soc. 40 (1989), 327-346.

22. E. Luft, The two-sided closed ideals of the algebra of bounded linear operators of a Hilbert space, Czech. Math. J. 18 (1968), 595-605.

23. T. W. PALmer, Banach algebras and the general theory of ${ }^{*}$-algebras, vol. 1 (Cambridge University Press, 1994).

24. A. Pietsch, Operator ideals (North-Holland, Amsterdam, 1980).

25. H. Porta, Ideaux bilatères de transformations linéaires continues, C. R. Acad. Sci. Paris A 264 (1967), 95-96.

26. E. SAKSMAN AND H.-O. Tylli, Weak compactness of multiplication operators on spaces of bounded linear operators, Math. Scand. 70 (1992), 91-111.

27. T. Schlumprecht, A complementably minimal Banach space not containing $c_{0}$ or $\ell_{p}$, in Seminar Notes in Functional Analysis and Partial Differential Equations, Baton Rouge, LA, 1992.

28. E. TARAFDAR, Improjective operators and ideals in a category of Banach spaces, J. Aust. Math. Soc. 14 (1972), 274-292.

29. P. Volkmann, Operatoralgebren mit einer endlichen Anzahl von maximalen Idealen, Studia Math. 55 (1976), 151-156.

30. R. J. Whithey, Strictly singular operators and their conjugates, Trans. Am. Math. Soc. 113 (1964), 252-261.

31. B. Yood, Difference algebras of linear transformations on a Banach space, Pac. J. Math. 4 (1954), 615-636. 